\title{
3D Bioprinted Implants for Cartilage Repair in Intervertebral Discs and Knee Menisci
}

\author{
Kalindu Perera ${ }^{1 \dagger}$, Ryan Ivone ${ }^{1 \dagger}$, Evelina Natekin ${ }^{2}$, Cheryl. A. Wilga ${ }^{3,4 *}$, Jie Shen ${ }^{1,5 *}$ and \\ Jyothi U. Menon ${ }^{1,5 *}$ \\ ${ }^{1}$ Department of Biomedical and Pharmaceutical Sciences, College of Pharmacy, University of Rhode Island, Kingston, Rl, \\ United States, ${ }^{2}$ Department of Biological Sciences, University of Alaska Anchorage, Anchorage, AK, United States, ${ }^{3}$ Department \\ of Biological Sciences, University of Rhode Island, Kingston, Rl, United States, ${ }^{4}$ Department of Electrical, Computer and \\ Biomedical Engineering, University of Rhode Island, Kingston, RI, United States, ${ }^{5}$ Department of Chemical Engineering, University \\ of Rhode Island, Kingston, RI, United States
}

OPEN ACCESS

Edited by:

Zhen $\mathrm{Li}$,

AO Research Institute, Switzerland

Reviewed by:

Yasuhiko Tabata,

Kyoto University, Japan

Feng-Huei Lin,

National Taiwan University, Taiwan

${ }^{*}$ Correspondence: Cheryl. A. Wilga

cwilga@uri.edu Jie Shen

jie_shen@uri.edu Jyothi U. Menon

jmenon@uri.edu

${ }^{t}$ These authors have contributed equally to this work

Specialty section:

This article was submitted to Tissue Engineering and Regenerative Medicine,

a section of the journal Frontiers in Bioengineering and

Biotechnology

Received: 05 August 2021 Accepted: 30 September 2021 Published: 22 October 2021

Citation:

Perera K, Ivone R, Natekin E, Wilga CA, Shen J and Menon JU (2021) 3D Bioprinted Implants for Cartilage Repair in Intervertebral Discs and Knee Menisci.

Front. Bioeng. Biotechnol. 9:754113. doi: 10.3389/fbioe.2021.754113
Cartilage defects pose a significant clinical challenge as they can lead to joint pain, swelling and stiffness, which reduces mobility and function thereby significantly affecting the quality of life of patients. More than 250,000 cartilage repair surgeries are performed in the United States every year. The current gold standard is the treatment of focal cartilage defects and bone damage with nonflexible metal or plastic prosthetics. However, these prosthetics are often made from hard and stiff materials that limits mobility and flexibility, and results in leaching of metal particles into the body, degeneration of adjacent soft bone tissues and possible failure of the implant with time. As a result, the patients may require revision surgeries to replace the worn implants or adjacent vertebrae. More recently, autograft - and allograft-based repair strategies have been studied, however these too are limited by donor site morbidity and the limited availability of tissues for surgery. There has been increasing interest in the past two decades in the area of cartilage tissue engineering where methods like 3D bioprinting may be implemented to generate functional constructs using a combination of cells, growth factors (GF) and biocompatible materials. 3D bioprinting allows for the modulation of mechanical properties of the developed constructs to maintain the required flexibility following implantation while also providing the stiffness needed to support body weight. In this review, we will provide a comprehensive overview of current advances in 3D bioprinting for cartilage tissue engineering for knee menisci and intervertebral disc repair. We will also discuss promising medical-grade materials and techniques that can be used for printing, and the future outlook of this emerging field.

Keywords: cartilage, bone repair, tissue engineering, 3D printing, bioprinting

\section{INTRODUCTION}

Treatment of cartilage injuries presents a significant challenge in modern orthopedics. Damage to the articular cartilage due to trauma, degenerative diseases or normal wear and tear affects everyone from children to the elderly. Poorly-healed cartilage defects cause serious and degenerative morbidities like osteoarthritis, which is the predominant cause of joint pain worldwide, affecting nearly 303 million people globally (Wei et al., 2021). The limited self-repair capabilities of the cartilage due to absence of 
vascularization, in which nutrients only reach chondrocytes via diffusion from the surrounding environment, and low chondrocyte density (comprising $1-5 \%$ of total cartilage) attributed to the high matrix to cell volume ratio, and the lack of operative and medical therapies that can significantly facilitate the healing process has led to an urgent need for new and durable treatments and grafting strategies to regenerate and repair these defects (Zhang et al., 2009; Akkiraju and Nohe, 2015; Dai and Gao, 2016).

Metal and plastic prosthetics that can recapitulate the surface of joints are currently the gold standard in cartilage and bone replacement. However, these are often rigid, limiting patient flexibility and mobility. Friction can lead to rapid wear of these implants, leading to damage and inflammation in adjacent tissues. Furthermore, the leaching of metal particles from the implants into the body can lead to adverse effects including tissue damage and poisoning (Prezyna, 2000; Sansone, 2013). Although current strategies to repair cartilage defects exist, including microfracture surgery, autologous chondrocyte implantation and osteochondral transplantation, these procedures have drawbacks such as high failure rates (25-50\% within 10 years) and reduced effectiveness among elderly patients (Lansdown et al., 2018). Furthermore, autologous chondrocyte transplantation is marred by shortage of chondrocyte sources, while microfracture surgery is limited by the development of fibrocartilage instead of natural hyaline cartilage in the region, which can worsen joint function (Zhang et al., 2019; Yang et al., 2020a; Wei et al., 2021). Other methods such as debridement and spongialization have been hindered by their clinical invasiveness and the high inherent risk of developing osteoarthritis (Zhang et al., 2019).

As an alternative to existing strategies, cartilage tissue engineering is being increasingly explored as a method of fabricating functional constructs that can facilitate the regeneration of joint cartilage. Among the many strategies under consideration, 3D bioprinting has emerged as a promising method that allows precise control over the properties of the construct including shape, architecture, mechanical strength and placement of cells and bioactive cues, to mimic native cartilage. In this review, we will explore in detail current technologies being developed for the repair of joint cartilage as well as $3 \mathrm{D}$ printing strategies and materials being used to improve the properties and functionality of 3D bioprinted constructs for the development of functional and durable implants. In particular, we will focus on high-risk, high-load bearing and motion-critical cartilage: namely, intervertebral discs and knee menisci, for which current treatment options (e.g., implants) do not offer satisfactory biological functionality.

\section{BIOMECHANICS OF JOINT CARTILAGE}

In the human body, the three main types of cartilage produced include hyaline cartilage, elastic cartilage, and fibrocartilage. Hyaline cartilage, the most common cartilage found in the body, functions to provide lubrication and load-bearing support for the articulating surfaces of bones in synovial

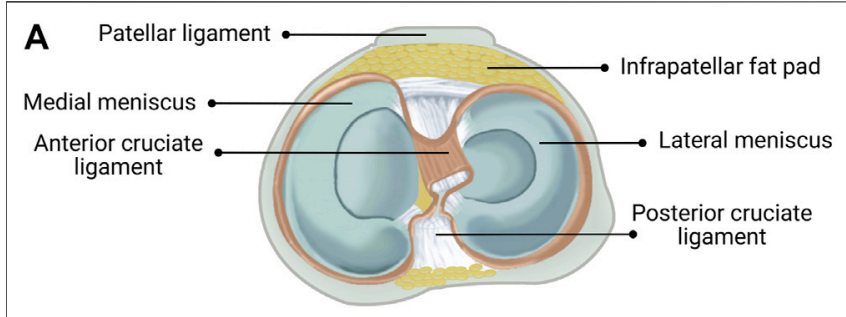

B

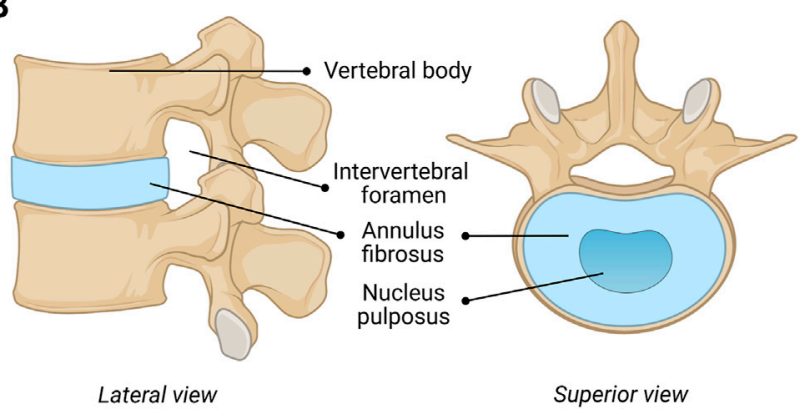

FIGURE 1 | Anatomical composition of the (A) human knee (including menisci) and (B) human intervertebral disc (IVD). Figure 1A reprinted with minor alterations from (Guo et al., 2015) under Creative Commons Public Domain Dedication waiver (creativecommons.org/publicdomain/zero/ 1.0)

joints, essential for joint movement, in addition to playing a key role in skeletal growth and development (Krishnan, 2018). Hyaline cartilage is comprised primarily of different types of collagen, as well as proteoglycans such as aggrecan, which provides compressive strength and load-bearing support for tissues due to intermolecular repulsive interactions ( $\mathrm{Ng}$ et al., 2003). Similar to hyaline cartilage, elastic cartilage is comprised mainly of collagen (type II) and proteoglycans. However elastic cartilage, which can be found in the ear and larynx also contains elastin fibers, allowing for increased flexibility while maintaining structural support (Chizhik et al., 2010). Knee menisci and intervertebral discs (IVDs) are comprised predominately of fibrocartilage, which exhibits high tensile strength due to the presence of thick, bundled, and highly ordered collagen (type I) fibers (Murphy et al., 2015).

\section{Knee Menisci}

The menisci are critical for maintaining the health and performance of the knee joint, providing nutrition, lubrication, shock absorption, load distribution, and stability of the joint (Fox et al., 2012; Kester et al., 2021). The semicircular medial- and circular lateral menisci are fibrocartilaginous wedges that are attached to the femur via multiple ligaments, as shown in Figure 1B. The menisci are concave at the femoral surface for articulation with the condyles and flat on the tibial surface to connect with the plateau. The outer border is thick and vascular allowing for firm attachment to the joint capsule, while the inner border is thin and avascular, allowing for correct orientation within the joint. The fibrochondrocytes (meniscal cells) maintain the dense extracellular matrix (ECM) and synthesize the collagen, 
proteoglycans, and other proteins embedded within (Makris et al., 2011). Tissue fluid comprises $65-70 \%$ of the total weight of menisci as most of the water is sequestered within proteoglycans (Herwig et al., 1984). The orientation of the collagen bundles in the center third region are primarily radially oriented suggesting that they function in compression, while those in the outer two-thirds are circumferentially oriented suggesting a tensile function (McDermott et al., 2008). In contrast, the collagen bundles are randomly oriented in the surface layer suggesting they function to decrease friction (McDermott et al., 2008).

Material properties of the shaped human meniscus is challenging due to the complexity of the structure. Three regions (anterior, middle, posterior) of fresh-frozen human meniscus were tested in compression at four strain rates $(3,6$, 9 , and $12 \%)$ at a physiologically relevant walking strain rate of $32 \%^{-\mathrm{s}}$ (Chia and Hull, 2008). Young's Modulus increased with greater strain and was higher in the anterior region $(1,048 \mathrm{kPa}$ at $12 \%)$ than the posterior region ( $329 \mathrm{kPa}$ at $12 \%$ strain), which is $8 x$ larger than at physiological equilibrium (Chia and Hull, 2008). Stiffness in the circumferential direction is greater than that in the axial and radial direction, which are similar, while thickness varies inversely with modulus (Lechner et al., 2000; Chia and Hull, 2008; Norberg et al., 2021). Meniscal biomechanics show that the lateral meniscus demonstrates greater mobility than the medial meniscus as the lateral meniscus has $9-11 \mathrm{~mm}$ of anteroposterior displacement and $11.2 \mathrm{~mm}$ of mediolateral displacement, while the medial meniscus exhibits only $2-3 \mathrm{~mm}$ of anteroposterior displacement and $5.1 \mathrm{~mm}$ of mediolateral displacement (Thompson et al., 1991).

\section{Intervertebral Discs}

The repair and regeneration of the cartilage of the human spine, which functions as the body's central support system and key to movement, has been the subject of intensive research and the results have been extensively described and summarized (Panjabi and White, 1980; Oxland, 2016). The human IVD functions to give a bipedal upright human spine motion, stability, and durability. The human IVD, shown in Figure 1A, consists of three components, including a multilayered outer ring of elastic collagen tissue fibers [annulus fibrosus (AF)], which is oriented at alternating angles (50-60 + degrees) that provides stability in torsion, compression and tension (Panjabi and White, 1980; Oxland, 2016). The concentric rings do not fully form complete circles in the posterior and posterolateral regions, which make these areas susceptible to herniation, fissures and failure (Panjabi and White, 1980; Oxland, 2016). The gelatinous elastic center (nucleus pulposus $(\mathrm{NuP})$ ) transmits stress and weight between vertebrae (Panjabi and White, 1980; Oxland, 2016). The NuP has semi-solid-like properties and therefore expands outward when compressed, which also expands the elastic fibers of the AF. This structure is connected to adjacent vertebrae by the Sharpey's fibers of the relatively flat cartilaginous end plates (CEP) of the vertebral bodies (Panjabi and White, 1980; Oxland, 2016). These three components, the AF, the NuP and the CEP are intimately integrated to function as a unit with the vertebrae above and below forming a spinal unit or motion unit that allows combinations of flexion, extension, lateral bending, and rotation of the spine at varying degrees depending on the location in the spine.

Mechanical properties of the human IVD are the topic of interest in many studies, and for good reason, given the debilitating results of their malfunction/degeneration (Markolf, 1974; Yoganandan et al., 1989; Iida et al., 2002; Stemper, 2010). While it had been believed that the $\mathrm{NuP}$ is mainly responsible for the elastic properties of the IVD, all components contribute significantly (Markolf, 1974). The compressive stiffness (E modulus: $19.5 \pm 4.1 \mathrm{MPa}$ in $28 \pm 8$ year old persons and $10.6 \pm 3.4 \mathrm{MPa}$ in $70 \pm 7$ year old persons) of the healthy IVD is 6-7 times higher than the tensile stiffness (E modulus: $2.9 \pm$ $0.8 \mathrm{MPa}$ in $28 \pm 8$ year old persons and $1.7 \pm 1 \mathrm{MPa}$ in $70 \pm$ 7 year old persons $3.3 \pm 2.1 \mathrm{MPa}$ ) (Iida et al., 2002; Stemper, 2010). Stiffness of the spine can vary depending on the spinal region (Shea et al., 1991). The loading of the IVD measured during normal activities was $0.1-0.5 \mathrm{MPa}$ and increased up to 2.3 MPa during lifting $20 \mathrm{~kg}$ weight (Wilke et al., 1999). Studies on sheep IVDs showed increased stiffness of IVD after cycled loading, which fully recovered after a period of unloading (Johannessen et al., 2004).

\section{CURRENT TECHNOLOGIES FOR JOINT CARTILAGE REPAIR}

Damage to- or degeneration of joint cartilage can lead to the development of debilitating arthritis, thereby impairing joint function (Abrams et al., 2013). In response to this, the number of research papers published on cartilage repair have nearly doubled in recent years, with the main focus being on replacement or regeneration of the knee meniscus and replacement of intervertebral discs (Abrams et al., 2013).

\section{Knee Cartilage}

The critical role of the meniscus in knee biomechanics and joint health is well known (Kester et al., 2021). Preservation of knee meniscus by repair, allograft transplantation and partial meniscectomy in the US is the current standard of care (Abrams et al., 2013; Kester et al., 2021). Over a 7 year period, the number of meniscectomies increased by $14 \%$ while the number of meniscus repairs increased by $100 \%$ (Abrams et al., 2013). Failure rates for meniscal repair were relatively low: $12 \%$ up to 1 year, $15 \%$ up to $2-3$ years, and $16.5-19 \%$ up to $4-6$ years post-repair (Ow, 2021). Meniscal repairs typically result in more revision surgeries, but remain a more effective long-term treatment than meniscectomies. Accordingly, while total meniscectomy can provide short-term relief and improve function, there remains a high risk of developing osteoarthritis over the long term (Abrams et al., 2013; Kester et al., 2021; Veronesi et al., 2021). The chondroprotective effects as well as the stabilizing and load-distributing function of the meniscus are thought to have led to the increase in the choice of repair over removal (Abrams et al., 2013). Advances in arthroscopic techniques, instrumentation, and postoperative care have also likely contributed to the rise in repairs (Abrams et al., 2013). 
Allograft transplants and implants show promise for restoring meniscal function. Transplant of meniscal allograft tissue to replace removed meniscus has shown encouraging results, however the availability of such tissue is limited, and can be subject to strict regulation in some countries (Veronesi et al., 2021). Meniscal scaffolds can stimulate repair and even regeneration of meniscal tissue, and is therefore an area of increasing research interest (Veronesi et al., 2021). Two acellular scaffolds are currently in clinical use, including Actifit (polyurethane-based) and collagen meniscus implant (CMI) (collagen-based). Both have demonstrated promising results in terms of moderate-to significant pain relief (measured on multiple scales) and improved movement/joint functionality over the mid-to long term (study durations lasting $<10$ years post-implantation) although regeneration is limited (Veronesi et al., 2021). These scaffolds remained biocompatible, while promoting limited meniscal healing, and therefore chondroprotection, which in turn decreased patient pain (Veronesi et al., 2021). Despite these early achievements, there has been a distinct lack of new meniscal implants (of any kind) approved for clinical use over the last decade.

\section{Intervertebral Discs}

Total disc replacement (TDR) and fusion of the spinal column have been used to treat degenerative disc disease for several years (Punt et al., 2008). Despite rising rates of fusion, procedures still come with concerns regarding failure to achieve a solid fusion mass (pseudarthrosis) and adjacent segment degeneration (Salzmann et al., 2017). TDR implantation rates have remained steady in the U.S. over the last decade, possibly due to issues with correct sizing and placement of the implant, the difficult nature of the surgery, lack of device selection, or fear of postoperative complications (Punt et al., 2008; Salzmann et al., 2017). The only TDR implants currently approved by the Food and Drug Administration (FDA) are metal based (U.S. Food and Drug Administration, 2020). THE PRODISC L TOTAL DISC REPLACEMENT -P050010/S020; U.S Food and Drug Administration (2015) activL $^{\circledR}$ Artificial DiscPatient Information -P120024. A006; Geisler, 2006).

Damaged IVDs of the spine can be fixed by replacing the disc with non-flexible material and then fusing the adjacent vertebrae using titanium plates, which results in reduced joint mobility (Rajaee et al., 2012). The number of patients having spinal fusion surgery increased from 203,053 to 442,776 annually from 1998 to 2014 (Sheikh et al., 2020). Despite providing temporary relief, adjacent spinal discs are often damaged due to increased stresses imposed on them as a result of lack of flexibility of fused vertebrae (Rajaee et al., 2012).

Artificial disc replacement has recently emerged as an alternative to fusion due to safer surgical procedures and better preservation of joint mobility (Park, 2015; Salzmann et al., 2017). However, total disc replacement (TDR) rates for intervertebral discs are low due to strict regulations for implant surgeries, demanding surgical techniques, low implant selection, and complications, requiring further surgery (Salzmann et al., 2017). Current TDR devices are composed of metal alloy plates sandwiching a plastic core, or a titanium mesh cage for bone infiltration that replaces the intervertebral disc, both of which can leach metal particles into the body and cause degeneration of relatively softer adjacent vertebrae and facets (FDA clears "first ever" 3D printed spine implant to treat of multiple injuries, 2018; Salzmann et al., 2017). One solution to preventing the development of adjacent segment disease is to preserve native biomechanics by replacing fusion techniques with motion sparing artificial discs. A new artificial cervical (SECURE-C TDR) metal disc has recently been developed to maintain physiologic motion, thereby reducing the risk of adjacent segment degeneration (McConnll, 2016).

There are currently three implants approved by the FDA for total disc replacement in the spine, including the Charite III Artificial Disc (DePuy Spine Inc., Raynham, MA), ProDisc-L (Synthes Spine, Paoli, PA) and the activL Artificial Disc (Aesculap Implant Systems, LLC), which are shown in Figures 2A,B, respectively (Salzmann et al., 2017). The Charité Artificial Disc consists of an ultra-high molecular weight polyethylene core that slides between the metallic alloy (containing cobalt, chromium, and molybdenum) endplates, with anchoring teeth on both top and bottom for attachment (Putzier et al., 2006). ProDisc-L consists of two metallic (containing cobalt and chromium) endplates and a plastic (ultra-high molecular weight polyethylene) ball-and-socket style center, with corrugated metal teeth on the top and bottom plates for attachment (U.S. Food and Drug Administration, 2020). THE PRODISC L TOTAL DISC REPLACEMENT-P050010/S020). The activL Artificial Disc is made of two cobalt-chromium alloy endplates and one polyethylene inlay. The plastic center is attached to the bottom endplate, and the top plate is designed to move over the center, allowing motion in all directions (U.S Food and Drug Administration, 2015) $\operatorname{activL}^{\circledR}$ Artificial DiscPatient Information-P120024. A006; Miller et al., 2016). There are three anchoring spikes on each of the top and bottom end plates. Additional materials used in intervertebral disc implants may include other cobalt-chromium alloys, stainless steel, titanium alloys, polyurethanes, and titanium alloy-ceramic composites (Pham et al., 2015).

Despite the successful use of metallic implants, there are still biomechanical-and toxicity concerns regarding wear debris (Salzmann et al., 2017). Wear reduces the lifetime of a prosthetic and leaves potentially harmful debris, potentially requiring the need for additional surgeries (Shankar and Kesavan, 2016). Several researchers have reported elevated levels of metal ions in the blood and urine of patients with metal-on-metal devices (Jacobs et al., 1996; Massè et al., 2003; Savarino et al., 2003). Postmortem studies have also found significant metal ion bioaccumulation in the liver, kidney, spleen, heart, and lymphatics of those patients outfitted with metallic implants (Urban et al., 2000). This metallosis is suspected to be an underreported or underdiagnosed issue, and has been shown to result in chronic inflammation, causing a host of unpleasant symptoms (nausea, cognitive impairment, hematological aberrations etc.) and more serious complications such as osteolysis or pseudotumors (Vaz et al., 2019). Further, more systemic and long-term impacts of circulating or accumulated metal ions is not completely understood, making 
A

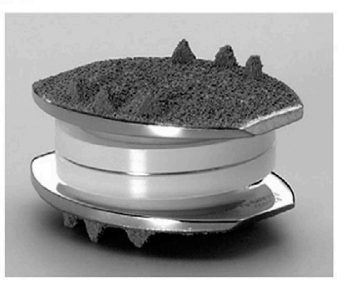

B

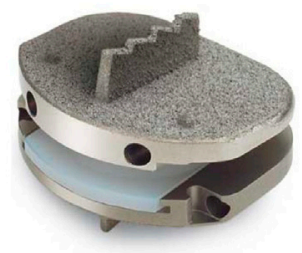

C

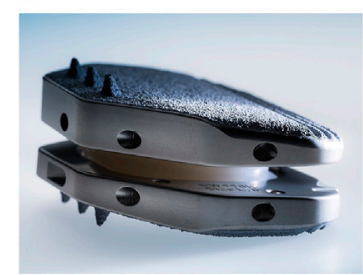

FIGURE 2 | Three intervertebral disc implants currently approved for use by the FDA including: (A) Charité III disc replacement (Depuy Spine) and (B) Prodisc-L (Centinel Spine) and (C) ActivL ${ }^{\circledR}$ artificial disc (Aesculap Implant Systems). Figures 2A,B reprinted with no alterations from (Kaner and Ozer, 2013 ) under Creative Commons Public Domain Dedication waiver (creativecommons.org/publicdomain/zero/1.0). Figure 2C (Aesculap's activL ${ }^{\circledR}$ Artificial Disc product image) used with permission from Aesculap Implant Systems, LLC, Center Valley, PA.

their avoidance desirable. The AcroFlex lumbar disc replacement is one example of a metallic implant that was discontinued after poor clinical outcomes (mechanical failures, osteolysis, etc.) (Germain and Tumialan, 2012; Meir et al., 2013). There is a need to fill this gap in available synthetic and non-metallic implants that exhibit some degree of flexibility and can conserve motion while providing sufficient load-bearing support.

\section{Overcoming Wear: The Evolution of Traditional Cartilage Repair}

Some attempts have been made to improve upon the wearresistance of materials used for the repair of cartilage injury. These encompass various nanocomposites, metal-on-polymer arrangements, ceramics and their composites, as well as woven materials, all with the goal of increasing wear resistance and decreasing wear debris and their physiological effects. One such example is oxidized zirconium (oxinium, possessing ceramic-like properties) with cross-linked polyethylene. Originally utilized for hip replacements, this technology has recently found its way to knee replacements, wherein the ends of the femur and tibia are capped with oxinium, with a polyethylene-based disc acting as the meniscus (Bhandari et al., 2012). The expected end-result is less metal-on-metal friction and wear. While touted as a replacement to older cobalt-containing setups, long-term studies have shown almost comparable (low) rates of wear, and no reduction in revision rates (Kim et al., 2012; Zou et al., 2020). Polymer through-wear followed by wear debris release has also been documented in some patients (Frye et al., 2021). Moreover, this technology involves surgery of even greater invasiveness owing to the complicated capping process. Oxinium debris has, however, shown lower inflammation elicitation than older cobalt counterparts (Rose et al., 2012). Other ceramics have also been used in this area, although mostly for hip arthroplasty. Ceramics have a low coefficient of friction and excellent wear resistance but are generally poor in terms of fracture-resistance owing to their brittleness, and sub-optimal load-bearing and flexibility capabilities (Yup Lee and Kim, 2010). Frequent squeaking of the joints during movement is a common patient complaint when using ceramic-on-ceramic joint replacements (Jarrett et al., 2009). A quite recent development has been the exploration of woven materials: being made of polymeric or hybrid polymer-natural fiber composites, these would have less harmful wear debris. Rodts et al. presented some interesting early work in this area, using laser welding to impart high wearresistance to their constructs, but-focusing entirely on wear resistance-did not provide any notable data with regard to mechanical properties or in vivo performance (Rodts et al., 2019). With no load-bearing substructure, it is difficult to envision these being applied successfully in cartilage or other constructs by themselves.

\section{D PRINTING FOR CARTILAGE ENGINEERING}

As a result of the limitations of current treatment options, there has been increased attention focused on cartilage and tissue engineering to overcome these limitations and facilitate joint regeneration. 3D printing is a popular type of additive manufacturing (AM) technology in which constructs are built in a layer-by-layer fashion, allowing for the design and production of patient-centric implants. 3D printing technology can be used to produce patient-personalized constructs (e.g., IVD's) in a time- and cost-effective manner, allowing for greater flexibility in terms of material selection, typically resulting in the production of more biocompatible constructs compared to metallic implants produced via traditional manufacturing methods (Lim et al., 2019). Various computer aided design (CAD) programs can be used to construct digital models, typically based on patient centric data obtained via computer tomography (CT) and magnetic resonance imaging (MRI) scans. These models can then be imported into software capable of editing and segmenting files, retaining only particular regions of interest. Lastly, files can be uploaded into a slicing software, where printing parameters such as number of layers and layer height are determined. This process can greatly improve the accuracy and translational potential of printed constructs.

Bioprinting is a subtype of $3 \mathrm{D}$ printing, in which the materials used in the $3 \mathrm{D}$ printing process contain cells and other biomaterials to produce a final construct. It is an emerging manufacturing technique used to develop tissue engineered constructs with very precise size and shape attributes, while maintaining excellent cell adhesion and proliferation abilities. 


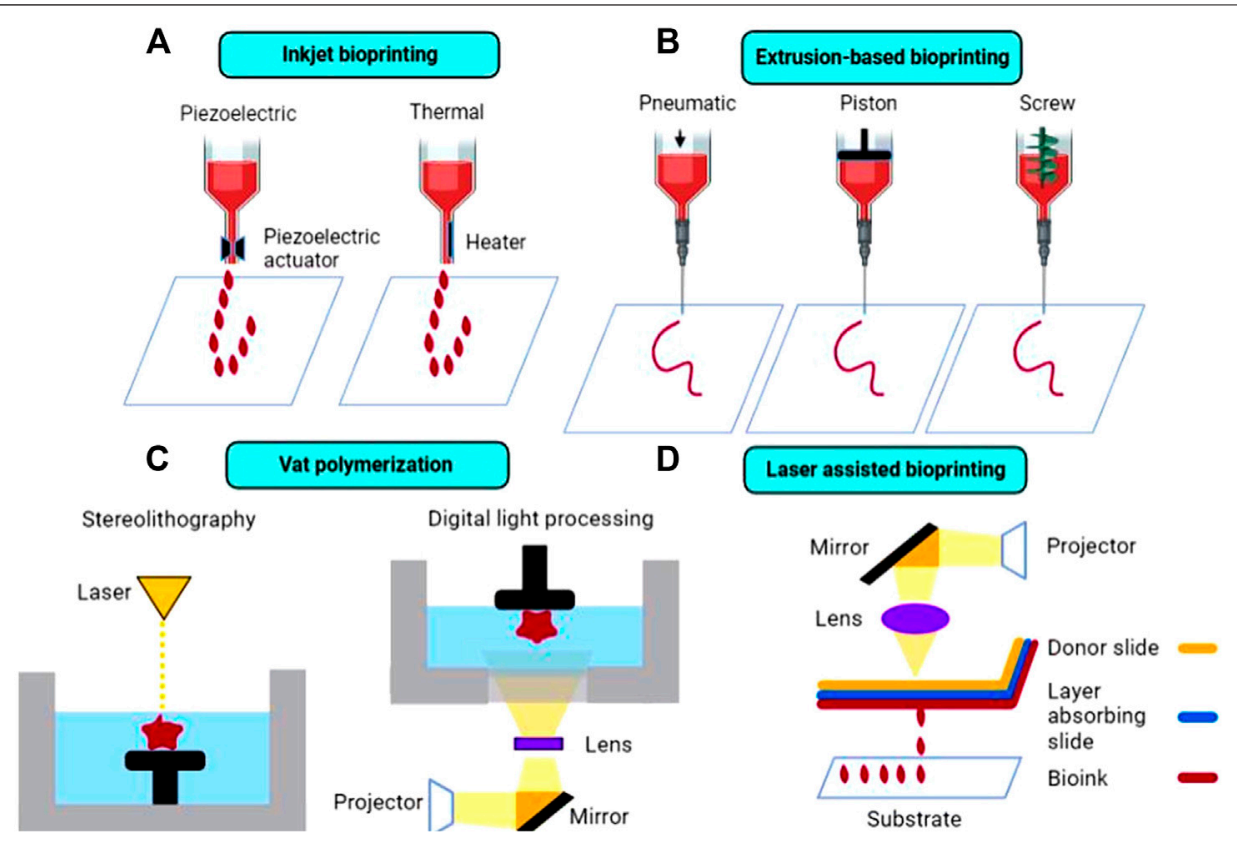

FIGURE 3 | Schematic diagram of the most common 3D bioprinting techniques in cartilage tissue engineering, including (A) inkjet bioprinting, (B) extrusion-based bioprinting, (C) vat polymerization, and (D) laser-assisted bioprinting.

Conventionally used metallic implants for meniscus and IVD repair fail to recapitulate the complex biological environment and mechanical structure of native tissue, while also presenting potential toxicity concerns via metal particle leaching in the body (Tavakoli et al., 2020). Overall biocompatibility of printed implants is intimately linked to the materials used in the printing process, which has been the focus of many review papers in recent years (Tappa and Jammalamadaka, 2018). In addition, traditional cartilage tissue engineering approaches result in scaffolds with a homogeneous distribution of chondrocytes or cartilage progenitor cells, and fail to recapitulate the complexity of native cartilage tissue (Daly and Kelly, 2019). Through meticulous material and printing parameter optimization, bioprinting allows for the fabrication of printed constructs demonstrating precise spatial and temporal control on the placement of cells and other bioactive substances (such as growth factors) while exhibiting similar chemical and mechanical properties as native tissue to better guide tissue formation, ideal for cartilage and tissue regeneration applications (Buj-Corral et al., 2018).

To produce 3D printed implants for cartilage repair, available $3 \mathrm{D}$ bioprinting techniques include inkjet bioprinting, extrusionbased bioprinting, vat polymerization (VP), and laser assisted bioprinting (LAB). Currently, inkjet and extrusion-based printers remain the most common types of printers used for cartilage tissue engineering applications.

\section{Inkjet Bioprinting}

Inkjet printing is a type of powder bed printing technology in which binder droplets, containing cells and other biomaterials (such as growth factors) are dispensed through overhead printheads and deposited onto a substrate, as shown in Figure 3A (Shirazi et al., 2015). Inkjet printing is advantageous compared to other traditional bioprinting techniques due to the capability to produce constructs in a cost-effective and high throughput manner (Li et al., 2020). For example, Daly et al. used an inkjet printing approach to produce stratified cartilage tissue by developing a bioink consisting of mesenchymal stem cells (MSC), chondrocytes and either pluronic or gelatin methacrylate (GelMA) and injecting this bioink into polymeric microchambers to guide cell growth and more representatively mimic native cartilage tissue (Daly and Kelly, 2019). However, the droplet ejection process, which can be achieved either via thermal or piezoelectric actuation, can be detrimental to maintaining adequate cell viability in printed constructs due to incurred shear and thermal stresses leading to cell death ( $\mathrm{Li}$ et al., 2016). In addition, depending on bioink viscosity, nozzle clogging can occur, preventing a smooth print from being achieved. Cui et al. (2014) modified an HP Deskjet 500 thermal inkjet printer, allowing it to print a photopolymerizable bioink containing human chondrocytes suspended in poly (ethylene glycol) diacrylate (PEGDA), suitable for cartilage tissue engineering applications.

\section{Extrusion-Based Bioprinting}

Traditionally, extrusion-based 3D printing, including fused deposition modeling (FDM) is accomplished by feeding a solid, thermoplastic filament through a high temperature nozzle, in which the material is then continuously extruded and deposited onto a lower temperature build plate, facilitating material solidification (Long et al., 2016). Despite 
allowing for rapid and efficient prototyping, FDM is not suitable for thermolabile components, including cell and proteins which can degrade under high temperatures. In addition, this method is only suitable for printing using solid filaments and is not capable of printing liquid cell suspensions. Thus, commercial bioprinters have been developed in recent years to overcome these obstacles. Extrusion-based commercial bioprinters rely on either pneumatic, piston, or screw-driven configurations to achieve continuous bioink extrusion, as shown in Figure 3B. In pneumatic-based extrusion, air pressure provides the main driving force for bioink dispensing, whereas in piston and screw-driven extrusion, vertical rotational forces are exerted on the bioink resulting in extrusion (Derakhshanfar et al., 2018). It is important to note that certain configurations, such as screw-driven extrusion, may negatively impact cell viability due to large pressure drops that occur along the nozzle (Ozbolat and Hospodiuk, 2016). Commercial bioprinters are typically considerably more expensive than traditional 3D printers, which may present a significant barrier to entry for some researchers interested in bioprinting, although more costeffective options have become available. Thus, some researchers have modified existing extrusion-based printers, enabling them to print liquid bioinks. For example, Gantumur et al. (2020) developed an alginate-based bioink containing fibroblast cells that can be printed at room temperature using a modified Prusi i3 3D printer via an enzyme-mediated hydrogelation method. Cell viability was similar in printed and non-printed hydrogels (54.1\% in printed vs $50.4 \%$ in non-printed after 1 day of culture), showing that this printing process did not adversely impact the fate of the cells and demonstrating the feasibility of this technique for bioprinting applications.

\section{Vat Polymerization Bioprinting}

Unlike extrusion-based printing, VP techniques rely on the layerby-layer solidification of liquid photopolymerizable resin, containing photopolymerizable monomer(s) and photoinitiator(s), as shown in Figure 3C. A build plate moves along the $\mathrm{z}$-axis inside a resin-containing vat. Upon exposure to a specific wavelength of light (dependent on resin components), the resin polymerizes and solidifies (Martinez et al., 2017). Layer adherence/formation is facilitated by unreacted monomers in the previous layer polymerizing upon light exposure. Stereolithography (SLA) and digital light processing (DLP) are the two main types of VP techniques, with the former using a laser beam and the latter using UV light from a projector to cure the resin. VP printing can overcome limitations inherent to other printing techniques, such as avoiding physical stresses imposed on bioinks (e.g., extrusion-based printing), which can ultimately lead to improved cell viability while maintaining high print resolution of final constructs (Kadry et al., 2019). Grogan et al. (2020) developed scaffold-free cartilage tissue constructs via a Regenova bioprinter in which microspheroids, held in place by a microneedle array, eventually fuse together to form neotissues. $\mathrm{Li}$ et al. (2017) combined 3D scanning technology with VP-based 3D printing (modified Bio-Architect, Regenovo) to more accurately replicate bone and cartilage defects, using either an alginate- or a hyaluronic acid (HA) based photopolymerizable hydrogel platform. Despite the advantages, concerns regarding photopolymer biocompatibility remains an issue, as unreacted resin components can present cytotoxicity issues at certain concentrations (Sabnis, 2010; Choi and Cha, 2019; Nguyen et al., 2019).

\section{Laser Assisted Bioprinting}

$\mathrm{LAB}$ is a technique in which a pulsed laser source (UV or nearUV wavelengths) is focused through a donor slide onto an absorbing layer, causing immediate vaporization and resulting in an area of high vapor pressure, facilitating droplet formation of the bioink layer underneath, which then deposits onto a substrate below (Figure 3D; Li et al., 2016). Printing via LAB circumvents issues typically encountered in traditional bioprinting processes, such as thermal and shear stresses that often lead to lowered cell viability in printed constructs. In addition, high resolution constructs and enhanced cellular organization can be achieved via LAB through optimization of ink droplet characteristics, such as ink viscosity and laser energy (Guillotin et al., 2013). However, LAB is a relatively time-consuming and expensive bioprinting method compared to conventional bioprinting techniques. Keriquel et al. (2017) developed a clinically feasible in situ forming collagen-nano hydroxyapatite (HAp) composite bioink containing mesenchymal stromal cells suitable for bone defect repair applications using a LAB technique. The LAB setup consisted of a near-infrared pulsed laser beam coupled to a scanning mirror, which focused the laser beam onto the absorbing layer (containing a thin layer of bioink), leading to droplet generation and deposition.

\section{BIOINKS}

Materials used to successfully produce bioprinted knee menisci and IVD's need to exhibit certain characteristics, such as biocompatibility to minimize immune response in the body; similar mechanical properties as native cartilage tissue to provide support to high load bearing regions such as in meniscus repair; and capability of promoting cell adhesion and proliferation, leading to cartilage tissue regeneration (Ahmed et al., 2019). Bioink components such as transforming growth factor beta-3 (TGF- $\beta 3$ ) and bone morphogenetic protein- 6 (BMP-6), can bind to cellular receptors, leading to cell differentiation and proliferation (Roseti et al., 2018). Daly et al. (2016) investigated properties of extrusion-based printing and the in vitro cartilage development achievable via the use of common bioinks, containing MSC and TGF- $\beta 3$, including agarose, alginate, GelMA and polyethylene glycol methacrylate $\left(\mathrm{PEGMA}\right.$, BioINK $\left.^{\mathrm{TM}}\right)$. Results demonstrated that agarose and alginate (which lack cell binding motifs) better supported the development of hyaline-like cartilage, while GelMA and PEGMA-based bioinks (which exhibit natural cell binding motifs and can promote cell spreading) were more suitable for fibrocartilaginous tissue development, illustrating that bioink composition plays a key role in determining cell phenotype. In addition, incorporation of polycaprolactone (PCL) fibers allowed for tailored mechanical properties to make printed constructs 
TABLE 1 | Examples of cartilage tissue constructs printed using different bioprinting techniques.

\begin{tabular}{|c|c|c|c|c|}
\hline Type of bioprinting & Cell type & Bioink materials & Cell viability & Ref \\
\hline \multirow[t]{3}{*}{ Inkjet } & Chondro-cytes & PEGDA & $90 \%$ & Cui et al. (2014) \\
\hline & MSC & PEGDMA & $>80 \%$ & Gao et al. (2015a) \\
\hline & & GelMA & & \\
\hline \multirow[t]{6}{*}{ Extrusion-based } & CPC & Alginate & Up to $89 \%$ & Yu et al. (2013) \\
\hline & Chondro-cytes & HA alginate & $>85 \%$ & Antich et al. (2020) \\
\hline & & PLA scaffold & & \\
\hline & Chondro-cytes & Alginate & $85-97 \%$ & Kundu, (2013) \\
\hline & & TGF $\beta$ & & \\
\hline & & PCL scaffold & & \\
\hline \multirow[t]{4}{*}{ Vat polymerization } & Chondro-cytes & GelMA & - & Chen et al. (2019) \\
\hline & & Decellularized cartilage ECM & & \\
\hline & Chondro-cytes & GelMA & Up to $95 \%$ & Lam et al. (2019) \\
\hline & & HAMA & & \\
\hline \multirow[t]{3}{*}{ Laser-assisted } & Mesench-ymal stromal cells & Collagen & - & Keriquel et al. (2017) \\
\hline & & Nano-hydroxyapatite & & \\
\hline & MSC & Alginate & - & Gruene et al. (2010) \\
\hline
\end{tabular}

CPC, cartilage progenitor cells; ECM, extracellular matrix; GelMA, gelatin methacrylate; HA, hyaluronic acid; HAMA, methacrylated hyaluronic acid; MSC, mesenchymal stem cell; PEGDA, poly(ethylene glycol) diacrylate; PEGDMA, poly(ethylene glycol) dimethacrylate; PLA, polylactic acid; PCL, polycaprolactone; TGF- $\beta$, transforming growth factor- $\beta$.

more suitable for load-bearing applications (e.g., articular cartilage designed for joint movement) and improve their translational potential. Examples of cartilage tissue constructs fabricated via different bioprinting techniques with various types of bioinks are shown in Table 1 .

\section{Natural Polymers}

Natural polymers with excellent biocompatibility and biological activity are an ideal class (relative to synthetic polymers) of materials suitable for use in bioinks, as they are capable of supporting cell attachment and differentiation (Liu et al., 2018). Despite the inherent advantages, natural polymers typically exhibit weaker mechanical properties than synthetic polymers, which can lead to poor printability and hinder in vivo performance. Constructs produced using solely natural polymers are typically more prone to failure within the body, as their mechanical properties are often not sufficient to withstand forces exerted in vivo. Thus, addition of materials that exhibit enhanced mechanical properties to form composites, and optimization of material crosslinking are strategies that are often used to develop more suitable natural polymer-based bioinks (Peng et al., 2020).

Alginate is a polysaccharide originally derived from brown algae and is often used to produce constructs capable of mimicking ECM structure by crosslinking with calcium ions $\left(\mathrm{Ca}^{2+}\right)$. Alginate is a promising bioink material due to its biocompatibility and rapid gelation process, leading to suitable printability. Yu et al. (2013) produced tubular channels, designed to mimic native vasculature, via an extrusion-based printer using a sodium alginate based bioink containing cartilage progenitor cells, capable of achieving cell viability up to $89 \%$. To improve printability and cell proliferation ability of alginate-based bioinks, composite materials have been developed. For example, Antich et al. (2020) developed a HA-alginate composite bioink able to produce chondrocyte-laden constructs that exhibited improved mechanical properties (e.g., increased viscosity) leading to enhanced printability, while maintaining impressive cell viability $(>85 \%)$ immediately after printing, with similar cell viability up to 4 weeks later.

$\mathrm{HA}$ is a linear polysaccharide and natural glycosaminoglycan (GAG) that plays numerous roles in the human body, such as maintaining ECM structure and acting as a signaling molecule capable of interacting with many cell surface receptors (Dicker, 2014). HA is a biocompatible and biodegradable material and can be easily chemically modified to alter its biological functions, thereby making it an ideal material for cartilage tissue engineering applications. However, like other natural polymers, HA exhibits relatively weak mechanical properties, leading to decreased printability. Ouyang et al. (2016) developed a 3D printable modified HA-based hydrogel ink, relying on guest-host supramolecular assembly via adamantane and $\beta$-cyclodextrin coupled to HA, resulting in increased viscosity and storage modulus values for modified HA hydrogels. In addition, fibroblast cells were seeded onto printed hydrogels and displayed adequate cell adhesion, illustrating the potential for this material to be used for cartilage tissue engineering applications. Park et al. (2014) developed a bioprinted (inhouse extrusion based printer) multicompartment hydrogel platform designed to promote osteochondral tissue regeneration, comprised of a chondrocyte-encapsulated HA compartment and osteoblast-encapsulated collagen (type I) compartment, capable of maintaining good cell viability of both cell types.

Collagen is the most abundant protein in the human body, and has been used to produce bioinks that effectively mimic the ECM structure while promoting cell adhesion, proliferation, and migration (Somaiah et al., 2015). As with other natural polymers, collagen suffers from relatively weak mechanical properties. Rhee et al. (2016) developed a bioink consisting of high concentration $(>10 \mathrm{mg} / \mathrm{ml})$ pure collagen containing meniscal fibrochondrocytes, and demonstrated that fabricated constructs could not accurately recapitulate the mechanical properties of native meniscal tissue. Shim et al. (2016) developed a multilayered construct using a bioink comprised 
of modified HA (Cucurbit [6] uril and 1,6-diaminohexane (DAH)-conjugated HA) and pepsin-treated collagen (atelocollagen) containing human turbinate-derived mesenchymal stromal cells as well as additional biomaterials (e.g., BMP-2 and TGF- $\beta$ ) capable of inducing cartilage regeneration in an in vivo rabbit model.

Gelatin is a linear peptide produced from the denaturation of collagen. Gelation can occur via chemical/enzymatic reactions or physical crosslinking, which requires heating and subsequent cooling to produce a semi-solid gel (Bello et al., 2020). Gelatin is an attractive bioink material due to its biocompatibility, biodegradability, and ease of modification (e.g., GelMA), allowing for facile crosslinking and enhanced cellular interactions (Gungor-Ozkerim et al., 2018). However, crosslinking of pure gelatin can be slow and may result in printed constructs that are mechanically inferior to native tissue. To overcome these issues, Singh et al. (2019) developed a gelatin encapsulated composite bioink containing a silk blend (Bombyx mori and Philosamia ricini) designed to enhance bioink mechanical properties, leading to enhanced printing fidelity while promoting the growth and proliferation of chondrocytes for cartilage tissues regeneration applications. Bioinks have also been developed containing modified gelatin, such as GelMA, in which the degree of methacryloylation and GelMA concentration have been shown to play a key role in modulating overall mechanical properties, including viscosity and stiffness (Leucht et al., 2020).

Other natural polymers, such as silk fibroin and elastin have recently been used in bioinks to produce more physiologically relevant IVD components (i.e., annulus fibrosus) (Costa et al., 2019). Such constructs demonstrate tunable degradation profile and mechanical properties, while remaining biocompatible. On a similar note, Tamo et al. developed a 3D printable chitosan-based hydrogel reinforced with cellulose nanofibers to better address the needs of developing mechanically demanding tissue repair strategies, for use in IVD and menisci repair applications, amongst others (Kamdem Tamo et al., 2021).

\section{Synthetic Polymers}

Compared to natural polymers, synthetic polymers such as polycaprolactone (PCL), polyethylene glycol (PEG), and PEG derivatives, such as Pluronic, exhibit more suitable mechanical properties, and are thus better able to withstand the forces exerted during the bioprinting process (Liu and Wang, 2020). In addition, they can support the development of porous structures and microchannels, which serve to recapitulate the vasculature of native tissue. Typically, however, synthetic polymers lack the biological activity necessary for promoting cell growth and proliferation and are thus used primarily for their role in providing structural support for bioinks (Wu et al., 2011).

PCL is a slow-degrading biocompatible polyester that has been used previously in bone tissue scaffold fabrication, and other medical products such as suture and bone screws (Chen et al., 2015; Cho et al., 2019; Dwivedi et al., 2020). PCL is commonly used in bioprinting applications to enhance the mechanical properties of printed constructs. Previous studies have supported this notion, as constructs have been fabricated using

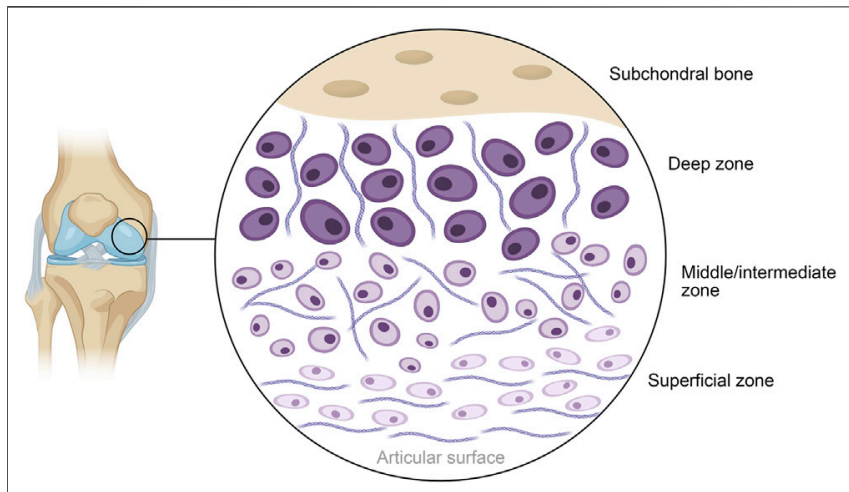

FIGURE 4 | Schematic diagram of the general structure of human articular cartilage, shown in the context of the knee. The deep zone contains hypertrophic chondrocytes interspersed with radially arranged collagen fibers, progressing to polymorphic chondrocytes and randomly arranged collagen in the middle layer. The superficial layer closest to the articular surface contains flattened chondrocytes packed in between horizontally arranged fibers of collagen.

PCL as a scaffold material demonstrating an increase in elastic moduli that more closely mimic native articular cartilage (Daly et al., 2016). Kundu (2013) developed a chondrocyte-containing alginate-based bioink able to be printed onto a PCL scaffold using an extrusion-based multi-head deposition system for cartilage tissue engineering. In this study, PCL was used as a support structure for the cell-laden alginate hydrogel, while facilitating cartilage tissue regeneration up to 14 days post-implantation, as demonstrated using a nude mice model.

PEG is a highly tunable, relatively inert synthetic polymer synthesized via the polymerization of ethylene oxide, that exhibits favorable mechanical properties suitable for cartilage tissue applications. Poloxamer, also known as pluronic, is a PEGbased block copolymer and is also commonly used in bioink development. PEG-based derivatives are attractive bioink materials due to their tunable gelation and favorable mechanical properties, both of which can be modulated by altering pluronic concentration (Geng et al., 2011). For instance, Ribeiro et al. (2018) investigated the printability of PEG/poloxamer-based bioink blends and demonstrated that bioink mechanical properties could be tuned by changing the PEG/poloxamer ratio, noticing that as this ratio increased, mechanical properties (i.e., yield stress, viscosity and storage modulus) decreased correspondingly. PEG and PEG derivatives have been combined with other biologically-active materials to facilitate cell adhesion and proliferation on printed constructs (Hutson et al., 2011; Rutz, 2015). For example, Armstrong et al. (2016) developed a pluronic-alginate composite bioink capable of producing constructs exhibiting a highly porous structure ideal for cell growth and nutrient transport, that supported the proliferation and differentiation of hMSCs over a 5-week period. In addition, photopolymerizable PEG-based materials, such as PEGMA, PEGDA and polyethylene-tetracrylate (PEGTA)-which can crosslink via light irradiation-have been developed and are commonly used in bioink formulations for their tunable mechanical properties 
and facile crosslinking abilities (Skardal et al., 2010). Gao et al. (2015b) developed a hMSC-encapsulated photopolymerizable PEGDMA-peptide bioink demonstrating good printability capable of producing scaffolds promoting bone-and cartilage regeneration.

\section{CELL-BASED 3D BIOPRINTING}

The earliest record of 3D bioprinting with cells being utilized for the regeneration or repair of cartilage is provided by the work of Cui et al. (2012). Fine spatial control and print resolution is highly useful when one considers the distinct structural zones that exist within articular cartilage, particularly the stratification by cell type. Chondrocyte organization, shape and expression patterns have been observed to vary markedly across three distinct layers: the superficial-, middle- and deep zones, as shown in Figure 4 (Akkiraju and Nohe, 2015). Alternatives to 3D bioprinting such as micromolding or other conventional techniques struggle to recreate these delicate features without significant and painstaking work, often with no guarantee of success. Bioprinting can allow cells with pre-induced expression profiles to be printed in distinct organizational patterns, densities, and depths, while other techniques with low control makes achieving local homogeneity in cell distribution a tough task. Tight spatial control also allows for the use of multiple polymer or matrix materials in complex patterns to achieve desired local-or global mechanical characteristics. This cellfriendly fabrication process allows cells to be seeded earlier on in the fabrication process, further improving fabrication times that have already been made shorter by bioprinting's automated nature. In all, these serve collectively to produce high quality, highly biomimetic cartilage structures.

\section{Cell-Based Bioprinting of Knee Cartilage}

Articular cartilage of the knee is one of the most commonly damaged type of cartilage in the human body, as a result of a variety of factors such as age, physical activity or diseases such as osteoarthritis, compounded by the joint's sheer complexity and load-bearing role (Gracitelli et al., 2015). As a result, most bioprinted cartilage applications have focused on regenerating the cartilage of the knee. Given that articular cartilage damage frequently co-occurs with lesions on the subchondral bone, a recent paper by Yang et al. (2020b) sought to produce an integrated, 3D bioprinted construct capable of regenerating both the damaged cartilage and the bone below it. To this end, they printed a layered composite consisting of sodium alginate and gelatin in the "cartilage" layer, and sodium alginate, gelatin and hydroxyapatite in the "bone" layer (the latter being an essential component of the bone regeneration process) with pore sizes of approximately $500 \mu \mathrm{m}$ (Kattimani et al., 2016). These inks were seeded with pre-differentiated osteogenic and chondrogenic bone marrow mesenchymal stem cells (BMSCs) prior to implantation on rabbit knee lesions for in vivo studies. The mechanical properties of this construct were inferior to that of natural cartilage, with a compressive strength of $11 \mathrm{MPa}$ after 6 months post-implantation $(30 \mathrm{MPa}$ in natural

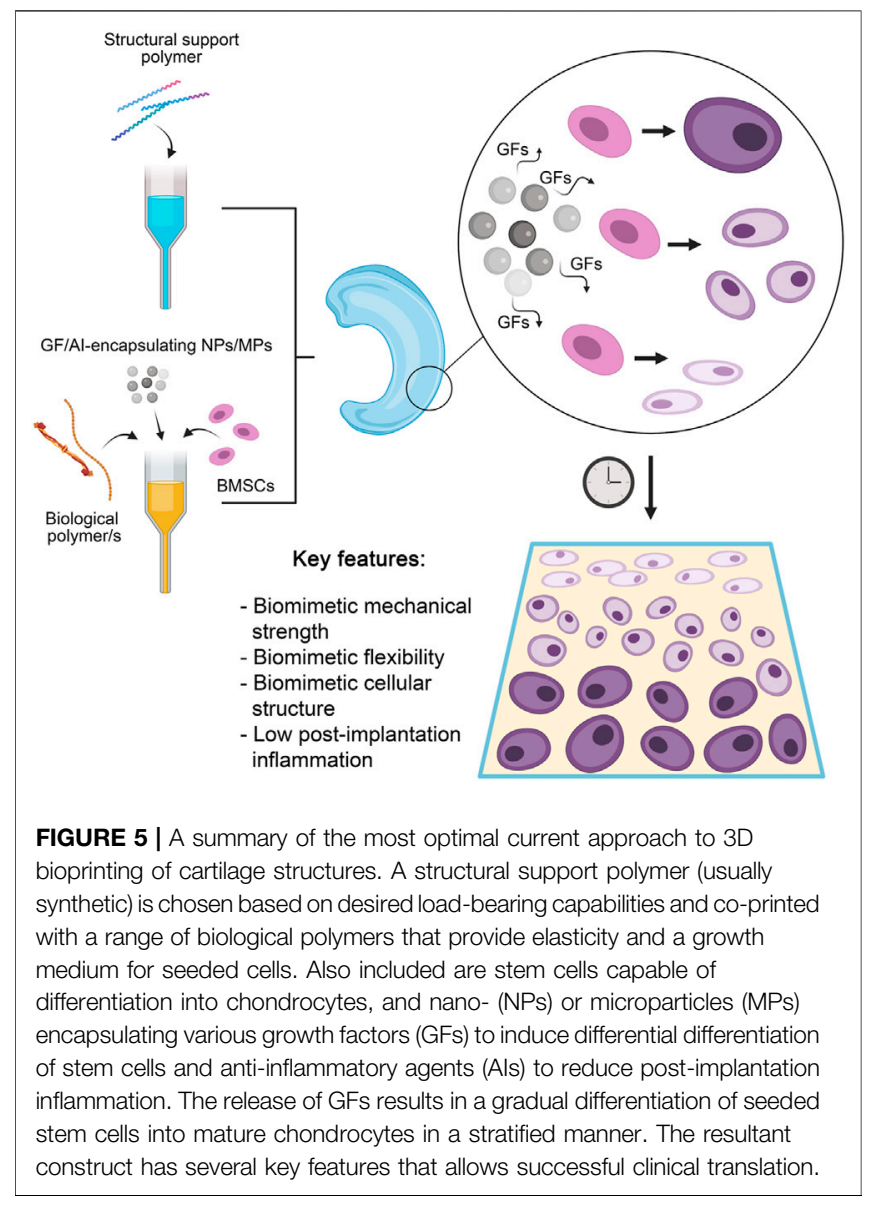

cartilage) and a maximum tolerable load of $183 \mathrm{~N}(480 \mathrm{~N}$ in natural cartilage), which may be attributed to the mechanical properties of the chosen bioink in this study. Despite this, cell viability in vitro was sufficiently high $(>70 \%$ by day 7$)$ and mechanical properties were significantly better than cell-less controls. Notably, the complete implants could produce hyaline-like cartilage with a still-disorganized structure and delicate links to the surrounding cartilage by 3 months postimplantation, and by 6 months, was histologically indistinguishable from the surrounding cartilage, although a small 'transition area' was visible. This stood in contrast to blank or even cell-less controls where repair either did not happen or was much slower and did not show the key integration with the surrounding cartilage or the subchondral bone. Another research group attempted a similar approach, printing a HAp-doped gelatin hydrogel $(10-50 \mu \mathrm{m}$ pore size) with human umbilical cord blood-derived mesenchymal stem cells (Huang, 2021). The construct was demonstrated to possess a compressive modulus of approximately $77 \mathrm{kPa}$, with HAp's role in lending compressive strength apparent in the lower compressive modulus of gelatin-only constructs $(70.5 \mathrm{kPa})$. This work demonstrated that HAp was able to induce a certain degree of chondrogenic differentiation as well, noting upregulation in the expression of chondrogenic markers aggrecan (ACAN) and Collagen Type II Alpha 1 Chain (COL2A1) from 
day 7 on, while levels of SRY-Box Transcription Factor 9 (SOX9) were elevated considerably from day $14 \mathrm{on}$. The authors did not, however, examine effects of the HAp on the construct's interaction with the subchondral bone, focusing instead on the HAp's ability to induce differentiation, provide strength/stiffness and their ability to act as excellent surfaces for chondrocyte growth and proliferation. In all, this integrative approach is one that merits inclusion in more works in the field of bioprinted cartilage.

The meniscus of the knee has also garnered the attention of 3D bioprinting, mostly due to the inability of current techniques of meniscus repair to address damage deeper in its structure (Guo et al., 2021). Luo et al. (2020) described the design and optimization of a bioink of gelatin, alginate and cellulose nanofibers loaded with rabbit fibrochondrocytes (rFCs). Several different formulations were tested, with pore size increasing proportional to both gelatin and cellulose content: low-gelatin alginate modified with cellulose nanofibers had pore sizes of approximately $180 \mu \mathrm{m}$ relative to the $300 \mu \mathrm{m}$ observed in high-gelatin alginate modified with cellulose. Their final construct was printed based on translated MRI imaging data and was thus patient-specific; it could maintain very high cell viabilities ( $>90 \%$ over 14 days) and displayed markedly high levels of collagen types II and X (Col II/X) in vitro. These are both indicators of healthy cartilage, the latter being indicative of the hypertrophic chondrocyte phenotype found deep in cartilage structure.

The incorporation of growth factors (GFs) and other molecules to initiate in situ differentiation of cells in bioprinted cartilage constructs (as illustrated in Figure 5) has been a growing area of interest. Several groups have published on this topic in recent years, examining in various ways how GF can influence cell-laden 3D bioprinted constructs (Henrionnet et al., 2020; Chawla et al., 2021). This has provided an impetus for the printing of constructs pre-loaded with GFs, in a major shift towards biological biomimicry over simple mechanical or morphological biomimicry. Sun et al. (2019) have been frequent contributors to the area, with one of their earlier works translating the findings of a genomics investigation into a viable cartilage regeneration/replacement strategy using $3 \mathrm{D}$ bioprinting. Having identified growth differentiation factor 5 (GDF5) as a chondroprotective agent capable of inducing chondrogenic differentiation in rabbit BMSCs, they encapsulated this agent within poly lactic-co-glycolic acid (PLGA) microparticles (MPs) which were then printed into the interfibrillar spaces of a PCL-based support structure, alongside a composite hydrogel made of gelatin, fibrinogen, hyaluronic acid and glycerol laden with rabbit BMSCs. More than $80 \%$ of encapsulated GDF5 was observed to be released from the microspheres within 60 days (in vitro), and cell proliferation increased exponentially in vitro in the 21-day period tested. Most notably, ultimate tensile strength (UTS) was very close to native knee cartilage, as implants exhibited a UTS value of $24 \mathrm{MPa}$ vs. the physiological UTS of $28 \mathrm{MPa}$. In vivo, GDF5 laden constructs were better able to produce hyaline-like neocartilage than constructs without GDF5 MPs, alongside higher GAG and Col II (also indicators of healthy native cartilage, and of chondrocyte phenotype) and displayed better cell spreading and proliferation. Continuing their work, the authors employed 3D bioprinting in the construction of a goat meniscus using a similar approach as above (Sun et al., 2021b). A hydrogel of the same type loaded with goat BMSCs and PLGA MPs containing connective tissue growth factor (CTGF) and (TGF $\beta 3$ ) were printed within a PCL scaffold. Of interest were the conversion of imaging data of a goat meniscus into a $3 \mathrm{D}$ model for the print and the differential distribution of the MPs within the meniscus (CTGF-containing MPs in the outer $1 / 3$, TGF $\beta 3$ MPs in the inner $2 / 3$ ) to stimulate differential BMSC differentiation, an improvement upon the indiscriminate differentiation in their previous work. Levels of SOX9 were observed to be elevated by $2 \mathrm{x}$ (CTGF MPs) to $3.5 \mathrm{x}$ (TGF $\beta 3 \mathrm{MPs}$ ) in vitro, relative to controls. Similar patterns of GAG and Col II vs Col I in the inner- and outer layers were also observed as in their previous work. In vivo, qualitative data showed that goats implanted with the complete construct had better 24-week mobility than those with control implants. Histological analysis of the implanted menisci in that period showed the expected native structure of an inner layer with an abundance of Col I-expressing fibroblast-like cells and the outer layer with Col II-expressing chondrocyte-like cells. Post-24 week implants displayed mechanical properties nearly identical to native cartilage, with statistically insignificant differences. Ultimate tensile strength, for instance, stood at approximately $29 \mathrm{MPa}$ in native cartilage vs. $28 \mathrm{MPa}$ in the implants; overall tensile modulus was higher in the implants by approximately $10 \mathrm{MPa}$. Radial and circumferential (both outer and inner layers) tensile modulus readings showed that implants lagged behind native cartilage by margins of less than $5 \%$. By contrast, Deng et al. employed human parathyroid hormone $(\mathrm{PH})$ to prevent chondrocyte hypertrophy in order to achieve and maintain hyaline-like properties (Deng, 2021). They achieved this using a biphasic, layered 3D bioprinted construct made of two distinct bioinks. The first was a mix of GelMA and $\mathrm{PH}$-conjugated SF loaded with rabbit articular cartilage cells, while the second was a mix of GelMA and methacrylated silk fibroin (MSF) loaded with BMSCs, whose pore sizes ranged from 150 to $300 \mu \mathrm{m}$. The SFcontaining construct was shown to possess a compressive elastic modulus of approximately $102.5 \mathrm{kPa} v s .211 .1 \mathrm{kPa}$ in the MSFcontaining constructs; compressive elastic strength was also higher when MSF was present $(260 \mathrm{kPa}$, a $4 \mathrm{x}$ increase over the SF-containing constructs). Significantly lower levels of collagen X and matrix metalloproteinase 13 (MMP13) were expressed by cells grown in the complete construct relative to $\mathrm{PH}$-negative controls. $\mathrm{PH}$ was also successful in engendering higher levels of Col II and ACAN in the cells, further confirming its role in maintaining hyaline-like phenotype. In vivo, the complete construct had filled lesions on rabbit knee cartilage by week 12 post-implantation. Yang et al. (2021) combined both GFs and MSC-recruiting aptamers in an interesting novel approach. Carbodiimide-mediated conjugation of HM69 aptamer was carried out on decellularized ECM, which was in turn dissolved in TGF 33 -containing GelMA. This bioink was then co-printed with PCL to produce a layered, dual-functional lattice structure with a heterogeneous macro- $(800-1,000 \mu \mathrm{m})$ and microporous $(80-200 \mu \mathrm{m})$ structure. The compressive modulus 
of this material was observed to be approximately $25 \mathrm{kPa}$, comparing favorably to expected in vivo physiological strain. While cells were later externally cultured on these constructs, what is of note was the ability of the HM69 to recruit adiposederived stem cells deeper into the construct both in vitro and in vivo. These GF-loaded, bioprinted constructs are of great use to this field as cell differentiation properties of printed constructs remains paramount to the success of biomimetic cartilage replacement/regeneration strategies. In particular, the inclusion of GFs in extended release micro-/nanoformulations into 3D bioprinted cartilage constructs circumvents a key stumbling block these constructs face during clinical translation-namely, the lack of long-term GF-based support once implanted (Francis et al., 2018).

Recognizing the need for anisotropy for clinical translation, Sun et al. (2020a) have also investigated the significance of construct pore sizes on vascularization and oxidative stress (which in turn influence cellular differentiation). Their design was a four-layer 3D bioprinted construct with gradients of both pore size and GFs. Using their BMSC-loaded, four-component hydrogel within a PCL support referred to previously, the construct was made such that the first layer was endowed with a pore size of $150 \mu \mathrm{m}$, which steadily increased to $750 \mu \mathrm{m}$ in the final, innermost layer. This gradient was designed to accommodate and encourage differentiation of rabbit BMSCs into the smaller flattened chondrocytes observed in the superficial zone of natural cartilage, then into the progressively larger polymorphic- and columnar chondrocytes of the middle zone, and finally, the hypertrophic chondrocytes of the deep zone. This approach was supplemented with PLGA microparticles encapsulating bone morphogenetic protein 4 (BMP4) in the innermost layer of the construct and transforming growth factor- $\beta 3$ (TGF $\beta 3$ ) in the outermost/superficial one.

In vitro, a clear gradient in the expression of COL2A1 and proteoglycan 4 (PRG4) (markers of the superficial zone of natural cartilage) was observed, decreasing steadily from superficial to deep layers (12x vs 1x for PRG4 and 9x vs 1x for COL2A1). Cells in this region were observed to be fibroblast-like. Conversely, the levels of Collagen Type X Alpha 1 Chain (COL10A1; cartilage deep zone marker) were 9 times higher in the deep layer (relative to superficial), resulting in clear chondrocyte hypertrophy. The authors also managed to match physiological mechanical properties (a Young's modulus of approx. $300 \mathrm{MPa} 12$ weeks post-implantation), maintain $>70 \%$ cell viability in vitro and confirm good functional capabilities/performance in vivo (with consistently higher histological scores and virtually indistinguishable integration with surrounding cartilage by week 24, relative to single-GF controls. Better microvessel ingrowth was also observed in the dual-GF constructs). These so-called 'dual stimuli' constructs also fared better than controls that either only had the pore size gradient alone or the growth factor gradient alone. Yet another work by the same group directed these anisotropic 3D bioprinting efforts at improving their meniscus construct mentioned previously (Sun et al., 2020b). Seeking again for better biomimicry, they repeated their goat meniscus work within a rabbit model, this time with the addition of microparticles carrying magnesium ions for enhanced vascularization of the superficial zone and skeletal muscle stem cells in place of BMSCs. Again, gradient cell differentiation was recapitulated, and the neovascularization of the superficial layer was observed to closely match the vascular pattern observed in natural menisci (confirmed with endothelial tube-forming assays, which showed the construct was highly capable of inducing the same). In vivo, the constructs matched natural cartilage in morphology, histological aspects, and mechanical properties by week 24 (the latter being almost identical to those seen in their earlier work). Anisotropy is a key feature of natural systems and replicating this is especially challenging in vitro. Nevertheless, these works discussed above have shown how $3 \mathrm{D}$ bioprinting can overcome this issue, and how established growth factor-loaded systems can be adapted for such. Moreover, vascularization of tissue constructs (vital particularly for menisci, which are naturally vascularized along one facet) has long been a significant obstacle for clinical translation of these technologies (Laschke and Menger, 2012; Francis et al., 2018). The work last discussed in this section has elegantly transcended this problem, combining anisotropy, spatial control of cell/ink placement and controlled release of GFs to set a benchmark for the field.

\section{Cell-Based Bioprinting of Intervertebral Discs}

Damage to the cartilage of the intervertebral discs (IVDs) can be particularly debilitating, given the key role the spine plays in support and movement. Recapitulating-both biologically and mechanically-the AF and $\mathrm{NuP}$ are important steps in producing replacements or regeneration strategies for IVDs. Composed of GAG and Col II, the NuP is a softer, elastic tissue at the core of the IVD, helping distribute pressure through it. The surrounding, harder AF is composed of collagen type I ( $\mathrm{Col} \mathrm{I}$ ), and acts as the tougher, load-bearing and shape-maintaining portion of the IVD (Sun et al., 2021a). Bioprinting efforts in this area (particularly in mimicking the AF) have been stymied by the lack of dedicated bioinks for IVDs, as others have already pointed out (Tavakoli et al., 2020). A notable example of a novel bioink for IVDs was presented by $\mathrm{Hu}$ et al., who combined thermoplastics and hydrogels in a tunable, 3D printed cartilage system using gellan gum-poly (ethylene glycol) diacrylate (GG-PEGDA) and PLA (Wu et al., 2018). A dualnozzle printing mechanism formed an outer ring of PLA to replicate the stiffer AF and GG-PEGDA hydrogels seeded with murine bone marrow mesenchymal stem cells (BMSCs) occupying the cavities of the PLA honeycomb substructure standing in for the softer NuP. A Young's modulus of $184 \mathrm{kPa}$ and a compressive strength of $55 \mathrm{kPa}$ was observed in the final construct. The near-total degradation of the PLA within 6 months, excellent cell viability ( $>90 \%, 7$ days) within the GG-PEGDA hydrogels and progressively high levels of F-actin observed through immunostaining after 7 days (indicating good cell spreading through the construct) were all signs that their bioprinted structure would be a highly biomimetic IVD replacement in vivo. Stevens et al. provides an overview of the development of GG-based bioinks, while a recent review by Pieri 
et al. provide a more comprehensive review of possible novel bioinks for IVD (Stevens et al., 2016; Pieri et al., 2020). Notwithstanding advances in this field, truly biomimetic IVD replacement/regeneration strategies are only beginning to be researched. Two recent pieces of work stand out for being on this cutting edge. Wu et al. (2021) recently outlined a PLA-based scaffold printed parallelly alongside GG-PEGDA/rat BMSCs, with the $\mathrm{NuP}$ and AF differentiated by the patterning of the printed strands, aiming to mimic the porosity and mechanical strength of the two. Of interest was the achievement of an excellent, physiological Young's modulus (remaining $>8 \mathrm{MPa}$ over 14 days). When combined with the $>80 \%$ in vitro cell viability 2 weeks post-seeding, the maintenance of $>75 \%$ disc height in vivo over 6 months and comparable levels of proteoglycan expression by the bioprinted implant as in the case of reimplanted natural IVDs over the same period, this is a clear sign that properly designed and -executed thermoplastichydrogel composites still hold real promise in this area. The authors did not, however, examine cellular differentiation along their NuP-AF axis, crucial for true biomimicry. Sun et al. (2021a) combined biomaterials, polymers, GFs, cells, and NPs in a complex milieu to form a highly biomimetic IVD replacement capable of inducing site-specific cell differentiation over time. Their NuP consisted of their characteristic gelatin-sodium alginate-hyaluronic acid hydrogel containing rat BMSCs and polydopamine (PDA) NPs decorated with TGF- $\beta 3$, while the AF consisted of the same hydrogel with BMSCs, and PDA NPs decorated with CTGF. Both were printed in a highly specific pattern within a 3D printed PCL scaffold and capped with cartilage endplates made of the same. Both GFs were found to be released steadily over more than 30 days, while good cell viability over this period in vitro predicted good differentiation of BMSCs into AF- and NuP-specific tissue in vivo. Most notably, these differentiations were observed to be clearly contained within the envisioned NuP/AF divide, with cells in the NuP portion expressing significantly higher GAG/Col II than those in the $\mathrm{AF}$, and cells in the $\mathrm{AF}$ expressing higher $\mathrm{Col} \mathrm{I}$ levels relative to those in the NuP. While the mechanical properties were not ideal (approaching, but not closely matching physiological compressive modulus), this is cause for excitement in this nascent field, showing how in situ differentiation along with the spatial control offered by $3 \mathrm{D}$ bioprinting can achieve remarkably biomimetic results.

One notable drawback of the cartilage constructs described so far is a phenomenon the authors themselves have highlighted- 3D bioprinted constructs, no matter how biomimetic, tend to elicit an immune response postimplantation that may last for weeks. Several recent works have outlined bioprinting strategies to solve this problem, albeit based more on traditional 3D printing supplemented with later cell seeding rather than direct bioprinting (Sun et al., 2019; 2020a). Zhu et al. (2020) described a PEGDA and decellularized ECM composite cartilage construct loaded with a natural anti-inflammatory agent (honokiol; $3^{\prime}, 5-\mathrm{Di}$ (prop-2-en-1-yl)[1,1'-biphenyl]-2,4'-diol). This was observed to suppress the release of pro-inflammatory cytokines including TNF- $\alpha$, IL- $1 \beta$ and IL- 6 in vitro (by approximately $2.5 \mathrm{x}, 3 \mathrm{x}$, and $2 \mathrm{x}$, respectively, all matching non-inflamed controls except in the case of IL-6). While levels of inflammation were not measured in vivo postimplantation, the use of mild natural compounds may certainly be helpful in forming bioprinted constructs that can manage inflammation without stressing the seeded cells. Lee et al. (2019) outlined a possible role for curcumin in bioprinted cartilage constructs (viz. a gelatin-curcumin bioink) but did not elaborate on any anti-inflammatory properties observed in their work. Indeed, natural antiinflammatory, analgesic compounds such as curcumin, gingerol, resveratrol and others hold much promise in this area- Buhrmann et al. (2020) provide a good recent review of these compounds and their myriad benefits in the context of cartilage tissue engineering in particular. Gong et al. (2020) too presented a method wherein anti-inflammatory IL-4 was printed into a GelMA hydrogel/PCL-hydroxyapatite support to achieve lower immune responses in their cartilage regeneration model. In vivo, they observed success in the form of higher histological scores and qualitatively better chondrocyte development post-implantation in the IL-4 loaded constructs compared to controls. This incorporation of anti-inflammatories-while not essential-is a relatively under-researched area in the field, and advances in this area will enhance clinical translation immensely. Figure 5 provides a summary and most optimal approach to cartilage bioprinting discussed herein.

\section{FUTURE OUTLOOK}

$3 \mathrm{D}$ bioprinting is a fast-moving field, and applications of this technology stand to benefit from this rapid pace of advancement. A recent development of interest is Samandari et al. report of multicompartmental bioprinting and its ability to successfully orient cells during printing (Samandari, 2021). The bioprinting of cartilage stands to gain from technologies such as this, as the spatial orientation of chondrocytes in constructs plays a significant role in in vitro and -vivo success. However, the need for such developments to be complemented by advances in polymer science and more significant contributions from nano- and microscale engineering must be recognized.

There is still much work to be done in the identification of polymer blends that accurately mimic the mechanical properties and biomechanics of natural cartilage, as well as blends that provide high load-bearing and wear-resistant capabilities. As menisci are responsible for stabilizing joints and acting as shock absorbers, mechanical properties remain one of the most important characteristics to consider when designing appropriate implants for meniscal repairs (Inyang and Vaughan, 2020). This is also of particular concern for IVDs, which differ in anatomy and function by location in the spinal column. For instance, in flexion and extension of the lumbar spine, anterior translation of one vertebra or the other should be $8 \%$ or less, while posterior translation should be $9 \%$ or less. Translation exceeding this is 
known as alteration of motion segment integrity (AOMSI). Additionally, angular motion of one lumbar vertebra to adjacent vertebra should allow no greater than 15 degrees difference within the first three lumbar units, L1/L2, L2/L3, or L3/L4 or no more than 20 degrees at L4/L5 and 25 degrees at L5/S1 (Keeney, 1984). This is a level of complexity that the field cannot yet achieve but must aim for eventually and must mostly be addressed using polymer science. Further, it has been demonstrated that mechanical properties, as well as mesh size (both of which can be impacted by polymer molecular weight) play a key role in modulating the viability and proliferation of chondrocyte-laden constructs (Lin et al., 2011). In addition, several studies have shown that modifications in polymer surface nanotopography (i.e., surface roughness) can lead to increased chondrocyte density and protein production due to enhanced protein binding on micro- and nano-rough surfaces (Storey and Webster, 2014). Advances in anti-inflammatory polymers or polymeric coatings may be of great use to this field as well-this is an active area of research for surgical implants in general, reviewed recently by both Sánchez-Bodón et al. and Lebaudy et al (Bridges and García, 2008; Al-Khoury et al., 2019; Lebaudy et al., 2021; Sánchez-Bodón et al., 2021). Recent work in this field has also evolved to incorporate decellularized extracellular matrix (dECM) from cartilage and IVD into 3D-printable bioinks to aid in guiding cell proliferation, attachment, and differentiation (Vernengo et al., 2020). For example, Kesti et al. (2015) developed a novel composite bioink comprised of gellan and alginate containing micronized BioCartilage, a commercially available extracellular matrix material native to articular cartilage, which supported chondrocyte proliferation and a favorable deposition of cartilage matrix proteins. While this particular bioink was developed for a broad range of tissue engineering applications, advancements like these may serve as a stepping-stone for the development of more finely-tuned bioinks to better address deficiencies in knee menisci and IVD repair strategies. The factors outlined above must all be considered when developing novel bioink formulations for this area of research.

Slow-release nano- or microformulations encapsulating growth factors and other signaling molecules to encourage proper cell differentiation, or those encapsulating agents such as anti-inflammatories or even anti-microbials to enhance postimplantation success are also of great interest to the field. Novel dual-action molecules tailored for cartilage repair like REG-O3 may be of interest to future researchers in this regard (Montjean et al., 2020). A possible way to integrate all this with traditional bioprinting may be to involve nano- or microscale fibers, such as carbon nanotubes (CNTs), that can lend structural support to constructs as well as releasing such agents from their core or their surface. Szymański (2020) provide an excellent review of recent tissue engineering work that has involved CNTs. Capable of selfassembly into nanostructures and acting as cell adhesion points or growth factor binding sites, peptide amphiphiles are also possible additives to cartilage bioinks that are already being explored in other tissue engineering approaches (Lewis et al.,
2020; Di Marzio et al., 2020). The next wave of bioink development should also most certainly focus on biomaterials that exhibit $4 \mathrm{D}$ bioprinting properties, consisting of materials that can be $3 \mathrm{D}$ printed, yet also respond to environmental stimuli (e.g., temperature, $\mathrm{pH}$, etc.) over a desired duration of time (Saska et al., 2021). For example, Betsch et al. (2018) developed a magnetically responsive iron nanoparticle-based bioink capable of producing constructs exhibiting alternating layers of aligned and random collagen fibers able to more accurately recapitulate the complex architecture of native articular cartilage, leading to enhanced collagen II production. Multilayered nano-/microparticles or -fibers can lend additional complexity to cartilage bioprinting by effecting fine control of chondrocyte differentiation and growth through temporally-controlled release of growth factors and other signaling molecules (Go et al., 2011; Asiri et al., 2021). Despite the availability of a considerable body of non-human in vivo data, 3D bioprinted cartilage constructs have-so far-not moved on to clinical trials. While fundamental research paves the way to more advanced applications, a field as nascent as this will eventually require clinical data for the refinement and evolution of the field, identifying shortcomings that exist in the in vitro development process that impact downstream clinical translation. In this light, it is heartening to note that such trials are now almost underway, with 3DBio Therapeutics' AuriNovo technology (3D bioprinted auricular cartilage for microtia) on the verge of recruiting trial subjects (NCT04399239). The technology is based on a proprietary bioink containing patient-derived (autologous) chondrocytes (AuriNovo for Auricular Reconstruction, 2020). This technology has been spurred in part by the introduction of personalized medicine to this field-a development rooted in the problem of possible implant rejection in a clinical setting as discussed earlier, and the autologous approaches needed to overcome it (Edri et al., 2019).

The need for printed constructs to encourage proper differentiation of seeded stem cells in the correct spatial orientation and patterning is another area that merits further investigation. The majority of works discussed in this review have opted for a static pore size, for instance. While there is evidence in literature that pore sizes of $100-300 \mu \mathrm{m}$ are optimal for cell differentiation and orientation within constructs, anisotropy in both this and in the release of growth factors, anti-inflammatory agents, anti-microbials etc. from nano/microparticles or -fibers may provide exciting new avenues of producing constructs that are more physiologically relevant, particularly when combined. This is highlighted by Sun and others' work (Zhang et al., 2014; Han et al., 2015; Naseri et al., 2016; Song et al., 2017; Sun et al., 2020a).

As tissue constructs go, cartilage has a relatively simple and straightforward structure to attempt to mimic than many other tissues in the human body. A successful convergence of new and improved polymers, precise bioprinting techniques focusing on anisotropy and dedicated "regions" of cartilage structure, optimized additives, and translation-friendly cell isolation, all complemented with regular feedback in the form of clinical data will define the direction this promising technology will take in the coming years. 


\section{AUTHOR CONTRIBUTIONS}

$\mathrm{KP}, \mathrm{RI}$, and EN wrote and prepared original draft; KP and RI edited and prepared final draft. CW, JS, and JUM reviewed and edited drafts and conceptualized this work.

\section{FUNDING}

We acknowledge funding support from the Rhode Island Institutional Development Award (IDeA) Network of Biomedical

\section{REFERENCES}

Abrams, G. D., Frank, R. M., Gupta, A. K., Harris, J. D., McCormick, F. M., and Cole, B. J. (2013). Trends in Meniscus Repair and Meniscectomy in the United States, 20052011. Am. J. Sports Med. 41, 2333-2339. doi:10.1177/0363546513495641

Ahmed, K. K., Tamer, M. A., Ghareeb, M. M., and Salem, A. K. (2019). Recent Advances in Polymeric Implants. AAPS PharmSciTech. 20, 300. doi:10.1208/ s12249-019-1510-0

Akkiraju, H., and Nohe, A. (2015). Role of Chondrocytes in Cartilage Formation, Progression of Osteoarthritis and Cartilage Regeneration. J. Dev. Biol. 3, 177-192. doi:10.3390/jdb3040177

Al-Khoury, H., Espinosa-Cano, E., Aguilar, M. R., Román, J. S., Syrowatka, F., Schmidt, G., et al. (2019). Anti-inflammatory Surface Coatings Based on Polyelectrolyte Multilayers of Heparin and Polycationic Nanoparticles of Naproxen-Bearing Polymeric Drugs. Biomacromolecules. 20, 4015-4025. doi:10.1021/acs.biomac.9b01098

Antich, C., de Vicente, J., Jiménez, G., Chocarro, C., Carrillo, E., Montañez, E., et al. (2020). Bio-Inspired Hydrogel Composed of Hyaluronic Acid and Alginate as a Potential Bioink for 3D Bioprinting of Articular Cartilage Engineering Constructs. Acta Biomater. 106, 114-123. doi:10.1016/j.actbio.2020.01.046

Armstrong, J. P. K., Burke, M., Carter, B. M., Davis, S. A., and Perriman, A. W. (2016). 3D Bioprinting Using a Templated Porous Bioink. Adv. Healthc. Mater. 5, 1724-1730. doi:10.1002/adhm.201600022

Asiri, A., Saidin, S., Sani, M. H., Al-Ashwal, R. H., and Ashwal, A. (2021). Epidermal and Fibroblast Growth Factors Incorporated Polyvinyl Alcohol Electrospun Nanofibers as Biological Dressing Scaffold. Sci. Rep. 11, 5634. doi:10.1038/s41598-021-85149-X

AuriNovo for Auricular Reconstruction (2020). Available at: https://clinicaltrials. gov/ct2/show/NCT04399239.

Bello, A. B., Kim, D., Kim, D., Park, H., and Lee, S.-H. (2020). Engineering and Functionalization of Gelatin Biomaterials: From Cell Culture to Medical Applications. Tissue Eng. B: Rev. 26, 164-180. doi:10.1089/ten.TEB.2019.0256

Betsch, M., Cristian, C., Lin, Y.-Y., Blaeser, A., Schöneberg, J., Vogt, M., et al. (2018). Incorporating 4D Into Bioprinting: Real-Time Magnetically Directed Collagen Fiber Alignment for Generating Complex Multilayered Tissues. Adv. Healthc. Mater. 7, 1800894-1800899. doi:10.1002/adhm.201800894

Bhandari, M., Pascale, W., Sprague, S., and Pascale, V. (2012). The Genesis II in Primary Total Knee Replacement: A Systematic Literature Review of Clinical Outcomes. The Knee. 19, 8-13. doi:10.1016/j.knee.2011.02.003

Bridges, A. W., and García, A. J. (2008). Anti-Inflammatory Polymeric Coatings for Implantable Biomaterials and Devices. J. Diabetes Sci. Technol. 2, 984-994. doi:10.1177/193229680800200628

Buhrmann, C., Honarvar, A., Setayeshmehr, M., Karbasi, S., Shakibaei, M., Valiani, A., et al. (2020). Herbal Remedies as Potential in Cartilage Tissue Engineering: An Overview of New Therapeutic Approaches and Strategies. Molecules. 25, 3075-3125. doi:10.3390/molecules25133075

Buj-Corral, I., Bagheri, A., and Petit-Rojo, O. (2018). 3D Printing of Porous Scaffolds With Controlled Porosity and Pore Size Values. Materials. 11, 1532-1618. doi:10.3390/ma11091532

Chawla, S., Turner, N., Terlizzo, M., and Heelan, K. (2021). Annular Atrophic Lichen Planus Induced by Anti-HER2 Antibodies. Australas. J. Dermatol. 62, 210-212. doi:10.1111/ajd.13501
Research Excellence from the National Institute of General Medical Sciences of the National Institutes of Health under grant number P20GM103430 (JUM), the Rhode Island Foundation Medical Research grant number 2797_20190601 (JS) and the University of Rhode Island Proposal Development Grant (JS, JUM).

\section{ACKNOWLEDGMENTS}

Figures 1B, 2, 3 and 4 were created with Biorender.com, with some post-modification by the authors.

Chen, P., Zheng, L., Wang, Y., Tao, M., Xie, Z., Xia, C., et al. (2019). DesktopStereolithography 3D Printing of a Radially Oriented Extracellular Matrix/ Mesenchymal Stem Cell Exosome Bioink for Osteochondral Defect Regeneration. Theranostics. 9, 2439-2459. doi:10.7150/thno.31017

Chen, T., Cai, T., Jin, Q., and Ji, J. (2015). Design and Fabrication of Functional Polycaprolactone. E-Polymers. 15, 3-13. doi:10.1515/epoly-2014-0158

Chia, H. N., and Hull, M. L. (2008). Compressive Moduli of the Human Medial Meniscus in the Axial and Radial Directions at Equilibrium and at a Physiological Strain Rate. J. Orthop. Res. 26, 951-956. doi:10.1002/ jor. 20573

Chizhik, S. A., Wierzcholski, K., Trushko, A. V., Zhytkova, M. A., and Miszczak, A. (2010). Properties of Cartilage on Micro- and Nanolevel. Adv. Tribology. 2010, 1. doi: $10.1155 / 2010 / 243150$

Cho, Y. S., Choi, S., Lee, S.-H., Kim, K. K., and Cho, Y.-S. (2019). Assessments of Polycaprolactone/Hydroxyapatite Composite Scaffold with Enhanced Biomimetic Mineralization by Exposure to Hydroxyapatite via a 3DPrinting System and Alkaline Erosion. Eur. Polym. J. 113, 340-348. doi:10.1016/j.eurpolymj.2019.02.006

Choi, G., and Cha, H. J. (2019). Recent Advances in the Development of NatureDerived Photocrosslinkable Biomaterials for 3D Printing in Tissue Engineering. Biomater. Res. 23, 18-27. doi:10.1186/s40824-019-0168-8

Costa, J. B., Silva-Correia, J., Ribeiro, V. P., da Silva Morais, A., Oliveira, J. M., and Reis, R. L. (2019). Engineering Patient-Specific Bioprinted Constructs for Treatment of Degenerated Intervertebral Disc. Mater. Today Commun. 19, 506-512. doi:10.1016/j.mtcomm.2018.01.011

Cui, X., Breitenkamp, K., Finn, M. G., Lotz, M., and D’Lima, D. D. (2012). Direct Human Cartilage Repair Using Three-Dimensional Bioprinting Technology. Tissue Eng. A. 18, 1304-1312. doi:10.1089/ten.tea.2011.0543

Cui, X., Gao, G., Yonezawa, T., and Dai, G. (2014). Human Cartilage Tissue Fabrication Using Three-Dimensional Inkjet Printing Technology. JoVE. 88, 1-5. doi:10.3791/51294

Dai, Y., and Gao, C. (2016). Cartilage Regeneration. Poly. Bio. Tiss. Regen. 21, 255-287. doi:10.1007/978-981-10-2293-7_9

Daly, A. C., Critchley, S. E., Rencsok, E. M., and Kelly, D. J. (2016). A Comparison of Different Bioinks for 3D Bioprinting of Fibrocartilage and Hyaline Cartilage. Biofabrication. 8, 045002-045010. doi:10.1088/17585090/8/4/045002

Daly, A. C., and Kelly, D. J. (2019). Biofabrication of Spatially Organised Tissues by Directing the Growth of Cellular Spheroids Within 3D Printed Polymeric Microchambers. Biomaterials. 197, 194-206. doi:10.1016/ j.biomaterials.2018.12.028

Deng, C., Yang, J., He, H., Ma, Z., Wang, W., Zhang, Y., et al. (2021). 3D BioPrinted Biphasic Scaffolds With Dual Modification of Silk Fibroin for the Integrated Repair of Osteochondral Defects. Biomater. Sci. 9, 4891-4903. doi:10.1039/d1bm00535a

Derakhshanfar, S., Mbeleck, R., Xu, K., Zhang, X., Zhong, W., and Xing, M. (2018). 3D Bioprinting for Biomedical Devices and Tissue Engineering: A Review of Recent Trends and Advances. Bioactive Mater. 3, 144-156. doi:10.1016/ j.bioactmat.2017.11.008

Di Marzio, N., Eglin, D., Serra, T., and Moroni, L. (2020). Bio-Fabrication: Convergence of 3D Bioprinting and Nano-Biomaterials in Tissue Engineering and Regenerative Medicine. Front. Bioeng. Biotechnol. 8, 1-11. doi:10.3389/fbioe.2020.00326 
Dicker, K. T., Gurski, L. A., Pradhan-Bhatt, S., Witt, R. L., Farach-Carson, M. C., and Jia, X. (2014). Hyaluronan: A Simple Polysaccharide With Diverse Biological Functions. Acta Biomater. 10, 1558-1570. doi:10.1016/ j.earlhumdev.2006.05.022

Dwivedi, R., Kumar, S., Pandey, R., Mahajan, A., Nandana, D., Katti, D. S., et al. (2020). Polycaprolactone as Biomaterial for Bone Scaffolds: Review of Literature. J. Oral Biol. Craniofac. Res. 10, 381-388. doi:10.1016/ j.jobcr.2019.10.003

Edri, R., Gal, I., Noor, N., Harel, T., Fleischer, S., Adadi, N., et al. (2019). Personalized Hydrogels for Engineering Diverse Fully Autologous Tissue Implants. Adv. Mater. 31, 1803895-1803899. doi:10.1002/adma.201803895

Edri, R.. (2018). FDA Clears "First Ever" 3D Printed Spine Implant to Treat of Multiple Injuries

Fox, A. J. S., Bedi, A., and Rodeo, S. A. (2012). The Basic Science of Human Knee Menisci. Sports Health. 4, 340-351. doi:10.1177/1941738111429419

Francis, S. L., Di Bella, C., Wallace, G. G., and Choong, P. F. M. (2018). Cartilage Tissue Engineering Using Stem Cells and Bioprinting Technology-Barriers to Clinical Translation. Front. Surg. 5, 1-12. doi:10.3389/fsurg.2018.00070

Frye, B. M., Laughery, K. R., and Klein, A. E. (2021). The Oxinium Arthrogram: A Sign of Oxidized Zirconium Implant Failure. Arthroplasty Today. 8, 103-109. doi:10.1016/j.artd.2021.02.001

Gantumur, E., Nakahata, M., Kojima, M., and Sakai, S. (2020). Extrusion-Based Bioprinting Through Glucose-Mediated Enzymatic Hydrogelation. Int. J. Bioprint. 6, 43-52. doi:10.18063/ijb.v6i1.250

Gao, G., Schilling, A. F., Hubbell, K., Yonezawa, T., Truong, D., Hong, Y., et al. (2015a). Improved Properties of Bone and Cartilage Tissue From 3D InkjetBioprinted Human Mesenchymal Stem Cells by Simultaneous Deposition and Photocrosslinking in PEG-GelMA. Biotechnol. Lett. 37, 2349-2355. doi:10.1007/s10529-015-1921-2

Gao, G., Yonezawa, T., Hubbell, K., Dai, G., and Cui, X. (2015b). InkjetBioprinted Acrylated Peptides and PEG Hydrogel With Human Mesenchymal Stem Cells Promote Robust Bone and Cartilage Formation With Minimal Printhead Clogging. Biotechnol. J. 10, 1568-1577. doi:10.1002/biot.201400635

Geisler, F. H. (2006). The CHARITE Artificial Disc: Design History, FDA IDE Study Results, and Surgical Technique. Clinical Neurosurgery. Editors G. McKhann II and G. A. Grant. (Philadelphia, PA: Lippincott Williams and Wilkins) 53, 223-228.

Geng, H., Song, H., Qi, J., and Cui, D. (2011). Sustained Release of VEGF from PLGA Nanoparticles Embedded Thermo-Sensitive Hydrogel in Full-Thickness Porcine Bladder Acellular Matrix. Nanoscale Res. Lett. 6, 312. doi:10.1186/1556276X-6-312

Germain, R., and Tumialan, L. M. (2012). Cervical and Lumbar Spinal Arthroplasty: Clinical Review. Am. J. Neuroradiol. 33, 1631-1641. doi:10.3174/ajnr.A2758

Go, D. P., Gras, S. L., Mitra, D., Nguyen, T. H., Stevens, G. W., Cooper-white, J. J., et al. (2011). Multilayered Microspheres for the Controlled Release of Growth Factors in Tissue Engineering. Biomacromolecules. 12, 1494-1503. doi:10.1021/ bm1014574

Gong, L., Li, J., Zhang, J., Pan, Z., Liu, Y., Zhou, F., et al. (2020). An Interleukin-4Loaded Bi-layer 3D Printed Scaffold Promotes Osteochondral Regeneration. Acta Biomater. 117, 246-260. doi:10.1016/j.actbio.2020.09.039

Gracitelli, G. C., Meric, G., Pulido, P. A., McCauley, J. C., and Bugbee, W. D. (2015). Osteochondral Allograft Transplantation for Knee Lesions after Failure of Cartilage Repair Surgery. Cartilage. 6, 98-105. doi:10.1177/ 1947603514566298

Grogan, S. P., Dorthé, E. W., Glembotski, N. E., Gaul, F., and D’Lima, D. D. (2020). Cartilage Tissue Engineering Combining Microspheroid Building Blocks and Microneedle Arrays. Connect. Tissue Res. 61, 229-243. doi:10.1080/ 03008207.2019.1617280

Gruene, M., Deiwick, A., Koch, L., Schlie, S., Unger, C., Hofmann, N., et al. (2011). Laser Printing of Stem Cells for Biofabrication of Scaffold-Free Autologous Grafts. Tissue Eng. C: Methods. 17, 79-87. doi:10.1089/ten.tec.2010.0359

Guillotin, B., Ali, M., Ducom, A., Catros, S., Keriquel, V., Souquet, A., et al. (2013). Laser-Assisted Bioprinting for Tissue Engineering. Biofabrication: Micro- and Nano-fabrication, Printing, Patterning and Assemblies. Editors G. Forgacs and W. Sun. (Waltham, MA: William Andrew), 95-118. doi:10.1016/B978-1-45572852-7.00006-8
Gungor-Ozkerim, P. S., Inci, I., Zhang, Y. S., Khademhosseini, A., and Dokmeci, M. R. (2018). Bioinks for 3D Bioprinting: An Overview. Biomater. Sci. 6, 915-946. doi:10.1039/c7bm00765e

Guo, W., Chen, M., Wang, Z., Tian, Y., Zheng, J., Gao, S., et al. (2021). 3D-Printed Cell-Free PCL-MECM Scaffold With Biomimetic Micro-Structure and MicroEnvironment to Enhance In Situ Meniscus Regeneration. Bioactive Mater. 6, 3620-3633. doi:10.1016/j.bioactmat.2021.02.019

Guo, W., Liu, S., Zhu, Y., Yu, C., Lu, S., Yuan, M., et al. (2015). Advances and Prospects in Tissue-Engineered Meniscal Scaffolds for Meniscus Regeneration. Stem Cell Int. 2015, 1-13. doi:10.1155/2015/517520

Han, K.-S., Song, J. E., Tripathy, N., Kim, H., Moon, B. M., Park, C. H., et al. (2015). Effect of Pore Sizes of Silk Scaffolds for Cartilage Tissue Engineering. Macromol. Res. 23, 1091-1097. doi:10.1007/s13233-015-3156-4

Henrionnet, C., Pourchet, L., Neybecker, P., Messaoudi, O., Gillet, P., Loeuille, D., et al. (2020). Combining Innovative Bioink and Low Cell Density for the Production of 3D-Bioprinted Cartilage Substitutes: A Pilot Study. Stem Cell Int. 2020, 1-16. doi:10.1155/2020/2487072

Herwig, J., Egner, E., and Buddecke, E. (1984). Chemical Changes of Human Knee Joint Menisci in Various Stages of Degeneration. Ann. Rheum. Dis. 43, 635-640. Available at: https://orthoinfo.aaos.org/en/treatment/artificial-diskreplacement-in-the-lumbar-spine/. doi:10.1136/ard.43.4.635

Huang, J., Huang, Z., Liang, Y., Yuan, W., Bian, L., Duan, L., et al. (2021). 3D Printed Gelatin/Hydroxyapatite Scaffolds for Stem Cell Chondrogenic Differentiation and Articular Cartilage Repair. Biomater. Sci. 9, 2620-2630. doi:10.1039/d0bm02103b

Hutson, C. B., Nichol, J. W., Aubin, H., Bae, H., Yamanlar, S., Al-Haque, S., et al. (2011). Synthesis and Characterization of Tunable Poly(ethylene Glycol): Gelatin Methacrylate Composite Hydrogels. Tissue Eng. Part A. 17, 1713-1723. doi:10.1089/ten.tea.2010.0666

Iida, T., Abumi, K., Kotani, Y., and Kaneda, K. (2002). Effects of Aging and Spinal Degeneration on Mechanical Properties of Lumbar Supraspinous and Interspinous Ligaments. Spine J. 2, 95-100. doi:10.1016/S1529-9430(02) 00142-0

Inyang, A. O., and Vaughan, C. L. (2020). Functional Characteristics and Mechanical Performance of PCU Composites for Knee Meniscus Replacement. Materials. 13, 1886. doi:10.3390/MA13081886

Jacobs, J. J., Skipor, A. K., Doorn, P. F., Campbell, P., Schmalzried, T. P., Black, J., et al. (1996). Cobalt and Chromium Concentrations in Patients With Metal on Metal Total Hip Replacements. Clin. Orthop. Relat. Res. 1, S256-S263. doi:10.1097/00003086-199608001-00022

Jarrett, C., Ranawat, A., Bruzzone, M., Blum, Y., Rodriguez, J., and Ranawat, C. (2009). The Squeaking Hip: A Phenomenon of Ceramic-on-Ceramic Total Hip Arthroplasty. J Bone Joint Surg Am, 91, 1344-1350. doi:10.2106/JBJS.F.00970

Johannessen, W., Vresilovic, E. J., Wright, A. C., and Elliott, D. M. (2004). Intervertebral Disc Mechanics Are Restored Following Cyclic Loading and Unloaded Recovery. Ann. Biomed. Eng. 32, 70-76. doi:10.1023/b: abme.0000007792.19071.8c

Kadry, H., Wadnap, S., Xu, C., and Ahsan, F. (2019). Digital Light Processing (DLP) 3D-Printing Technology and Photoreactive Polymers in Fabrication of Modified-Release Tablets. Eur. J. Pharm. Sci. 135, 60-67. doi:10.1016/ j.ejps.2019.05.008

Kamdem Tamo, A., Doench, I., Walter, L., Montembault, A., Sudre, G., David, L., et al. (2021). Development of Bioinspired Functional Chitosan/Cellulose Nanofiber 3d Hydrogel Constructs by 3d Printing for Application in the Engineering of Mechanically Demanding Tissues. Polymers. 13, 1663. doi: $10.3390 /$ polym 13101663

Kaner, T., and Ozer, A. F. (2013). Dynamic Stabilization for Challenging Lumbar Degenerative Diseases of the Spine: A Review of the Literature. Adv. Orthopedics. 2013, 1-13. doi:10.1155/2013/753470

Kattimani, V. S., Kondaka, S., and Lingamaneni, K. P. (2016). Hydroxyapatite-Past, Present, and Future in Bone Regeneration. Bone Tissue Regen. Insights. 7, BTRI.S36138-19. doi:10.4137/btri.s36138

Keeney, A. H. (1984). Guides to the Evaluation of Permanent Impairment, 2nd Ed. Am. J. Ophthalmol. 98, 659. doi:10.1016/0002-9394(84)90290-3

Keriquel, V., Oliveira, H., Rémy, M., Ziane, S., Delmond, S., Rousseau, B., et al. (2017). In Situ printing of Mesenchymal Stromal Cells, by Laser-Assisted Bioprinting, for In Vivo Bone Regeneration Applications. Sci. Rep. 7, 1-10. doi:10.1038/s41598-017-01914-x 
Kester, C. R., Caldwell, P. E., and Pearson, S. E. (2021). Lateral Meniscal Allograft Transplant: Dovetail Bone Bridge Preparation. Arthrosc. Tech. 10, e969-e973. doi:10.1016/j.eats.2020.11.008

Kesti, M., Eberhardt, C., Pagliccia, G., Kenkel, D., Grande, D., Boss, A., et al. (2015). Bioprinting Complex Cartilaginous Structures With Clinically Compliant Biomaterials. Adv. Funct. Mater. 25, 7406-7417. doi:10.1002/adfm. 201503423

Kim, Y.-H., Park, J.-W., and Kim, J.-S. (2012). Comparison of the Genesis II Total Knee Replacement With Oxidised Zirconium and Cobalt-Chromium Femoral Components in the Same Patients. The J. Bone Jt. Surg. Br. 94, 1221-1227. doi:10.1302/0301-620X.94B9.28854

Krishnan, Y. (2018). Cartilage Diseases. Matrix Biol. 71-72, 1-31. doi:10.1016/ j.matbio.2018.05.005.Cartilage

Kundu, J., Shim, J.-H., Jang, J., Kim, S.-W., and Cho, D.-W. (2013). An Additive Manufacturing-Based PCL-Alginate-Chondrocyte Bioprinted Scaffold for Cartilage Tissue Engineering. J. Tissue Eng. Regen. Med. 9, 1286-1297. doi:10.1002/term.1682

Lam, T., Dehne, T., Krüger, J. P., Hondke, S., Endres, M., Thomas, A., et al. (2019). Photopolymerizable Gelatin and Hyaluronic Acid for Stereolithographic 3D Bioprinting of Tissue-Engineered Cartilage. J. Biomed. Mater. Res. 107, 2649-2657. doi:10.1002/jbm.b.34354

Laschke, M. W., and Menger, M. D. (2012). Vascularization in Tissue Engineering: Angiogenesis Versus Inosculation. Eur. Surg. Res. 48, 85-92. doi:10.1159/ 000336876

Lebaudy, E., Fournel, S., Lavalle, P., Vrana, N. E., and Gribova, V. (2021). Recent Advances in Antiinflammatory Material Design. Adv. Healthc. Mater. 10, 2001373-2001420. doi:10.1002/adhm.202001373

Lechner, K., Hull, M. L., and Howell, S. M. (2000). Is the Circumferential Tensile Modulus Within a Human Medial Meniscus Affected by the Test Sample Location and Cross-Sectional Area? J. Orthop. Res. 18, 945-951. doi:10.1002/ jor.1100180614

Lee, M. J., Kim, S. E., Park, J., Ahn, G. Y., Yun, T. H., Choi, I., et al. (2019). Curcumin-Loaded Biodegradable Polyurethane Scaffolds Modified With Gelatin Using 3D Printing Technology for Cartilage Tissue Engineering. Polym. Adv. Technol. 30, 3083-3090. doi:10.1002/pat.4740

Leucht, A., Volz, A.-C., Rogal, J., Borchers, K., and Kluger, P. J. (2020). Advanced Gelatin-Based Vascularization Bioinks for Extrusion-Based Bioprinting of Vascularized Bone Equivalents. Sci. Rep. 10, 1-15. doi:10.1038/s41598-02062166-w

Lewis, J. A., Freeman, R., Carrow, J. K., Clemons, T. D., Palmer, L. C., and Stupp, S. I. (2020). Transforming Growth Factor $\beta-1$ Binding by Peptide Amphiphile Hydrogels. ACS Biomater. Sci. Eng. 6, 4551-4560. doi:10.1021/acsbiomaterials.0c00679

Li, J., Chen, M., Fan, X., and Zhou, H. (2016). Recent Advances in Bioprinting Techniques: Approaches, Applications and Future Prospects. J. Transl. Med. 14, 1-15. doi:10.1186/s12967-016-1028-0

Li, L., Yu, F., Shi, J., Shen, S., Teng, H., Yang, J., et al. (2017). In Situ repair of Bone and Cartilage Defects Using 3D Scanning and 3D Printing. Sci. Rep. 7, 1-12. doi:10.1038/s41598-017-10060-3

Li, X., Liu, B., Pei, B., Chen, J., Zhou, D., Peng, J., et al. (2020). Inkjet Bioprinting of Biomaterials. Chem. Rev. 120, 10793-10833. doi:10.1021/acs.chemrev.0c00008

Lim, W., Kim, B., and Moon, Y. L. (2019). Three-Dimensional Bioprinting for Bone and Cartilage Transplantation. Ann. Jt. 4, 7. doi:10.21037/aoj.2018.12.06

Lin, S., Sangaj, N., Razafiarison, T., Zhang, C., and Varghese, S. (2011). Influence of Physical Properties of Biomaterials on Cellular Behavior. Pharm. Res. 28, 1422-1430. doi:10.1007/s11095-011-0378-9

Liu, F., Chen, Q., Liu, C., Ao, Q., Tian, X., Fan, J., et al. (2018). Natural Polymers for Organ 3D Bioprinting. Polymers. 10, 1278-1326. doi:10.3390/polym10111278

Liu, F., and Wang, X. (2020). Synthetic Polymers for Organ 3D Printing. Polymers. 12, 1765. doi:10.3390/polym12081765

Long, J., Gholizadeh, H., Lu, J., Bunt, C., and Seyfoddin, A. (2016). Application of Fused Deposition Modelling (FDM) Method of 3D Printing in Drug Delivery. Curr. Pharm. Des. 23, 433-439. doi:10.2174/13816128226661610261

Luo, W., Song, Z., Wang, Z., Wang, Z., Li, Z., Wang, C., et al. (2020). Printability Optimization of Gelatin-Alginate Bioinks by Cellulose Nanofiber Modification for Potential Meniscus Bioprinting. J. Nanomater. 2020, 1-13. doi:10.1155/ $2020 / 3863428$

Makris, E. A., Hadidi, P., and Athanasiou, K. A. (2011). The Knee Meniscus: Structure-Function, Pathophysiology, Current Repair Techniques, and
Prospects for Regeneration. Biomaterials. 32, 7411-7431. doi:10.1016/ j.biomaterials.2011.06.037

Markolf, K. L., and Morris, J. M. (1974). The Structural Components of the Intervertebral Disc. J. Bone Jt. Surg. 56, 675-687. doi:10.2106/00004623197456040-00003

Martinez, P. R., Goyanes, A., Basit, A. W., and Gaisford, S. (2017). Fabrication of Drug-Loaded Hydrogels With Stereolithographic 3D Printing. Int. J. Pharmaceutics. 532, 313-317. doi:10.1016/j.ijpharm.2017.09.003

Massè, A., Bosetti, M., Buratti, C., Visentin, O., Bergadano, D., and Cannas, M. (2003). Ion Release and Chromosomal Damage From Total Hip Prostheses With Metal-On-Metal Articulation. J. Biomed. Mater. Res. 67B, 750-757. doi:10.1002/jbm.b.10070

McConnll, J. (2016). Motion Preservation at the Operative Level and the Incidence of Symptomatic Adjacent Segment Disease After Treatment With SECURE ${ }^{\circledR}$-C or ACDF. Spine J. 16, 189. doi:10.1016/j.spinee.2016.07.179

McDermott, I. D., Masouros, S. D., and Amis, A. A. (2008). Biomechanics of the Menisci of the Knee. Curr. Orthopaedics. 22, 193-201. doi:10.1016/ j.cuor.2008.04.005

Meir, A. R., Freeman, B. J. C., Fraser, R. D., and Fowler, S. M. (2013). Ten-Year Survival and Clinical Outcome of the AcroFlex Lumbar Disc Replacement for the Treatment of Symptomatic Disc Degeneration. Spine J. 13, 13-21. doi:10.1016/j.spinee.2012.12.008

Miller, L. E., Yue, J., and Garcia, R. (2016). The activL ${ }^{\circledR}$ Artificial Disc: A NextGeneration Motion-Preserving Implant for Chronic Lumbar Discogenic Pain. Med. Devices: Evidence Res. 9, 75-84. doi:10.2147/MDER.S102949

Montjean, R., Escaich, S., Paolini, R., Carelli, C., Pirson, S., Neutelings, T., et al. (2020). REG-O3 Chimeric Peptide Combining Growth Hormone and Somatostatin Sequences Improves Joint Function and Prevents Cartilage Degradation in Rat Model of Traumatic Knee Osteoarthritis. PLoS One. 15, e0231240-17. doi:10.1371/journal.pone.0231240

Murphy, M. K., Masters, T. E., Hu, J. C., and Athanasiou, K. A. (2015). Engineering a Fibrocartilage Spectrum Through Modulation of Aggregate Redifferentiation. Cell Transpl. 24, 235-245. doi:10.3727/096368913X676204

Naseri, N., Poirier, J.-M., Girandon, L., Fröhlich, M., Oksman, K., and Mathew, A. P. (2016). 3-Dimensional Porous Nanocomposite Scaffolds Based on Cellulose Nanofibers for Cartilage Tissue Engineering: Tailoring of Porosity and Mechanical Performance. RSC Adv. 6, 5999-6007. doi:10.1039/c5ra27246g

Ng, L., Grodzinsky, A. J., Patwari, P., Sandy, J., Plaas, A., and Ortiz, C. (2003). Individual Cartilage Aggrecan Macromolecules and Their Constituent Glycosaminoglycans Visualized via Atomic Force Microscopy. J. Struct. Biol. 143, 242-257. doi:10.1016/j.jsb.2003.08.006

Nguyen, A. K., Goering, P. L., Reipa, V., and Narayan, R. J. (2019). Toxicity and Photosensitizing Assessment of Gelatin Methacryloyl-Based Hydrogels Photoinitiated with Lithium Phenyl-2,4,6-Trimethylbenzoylphosphinate in Human Primary Renal Proximal Tubule Epithelial Cells. Biointerphases. 14, 021007-021008. doi:10.1116/1.5095886

Norberg, C., Filippone, G., Andreopoulos, F., Best, T. M., Baraga, M., Jackson, A. R., et al. (2021). Viscoelastic and Equilibrium Shear Properties of Human Meniscus: Relationships With Tissue Structure and Composition. J. Biomech. 120, 110343. doi:10.1016/j.jbiomech.2021.110343

Ouyang, L., Highley, C. B., Rodell, C. B., Sun, W., and Burdick, J. A. (2016). 3D Printing of Shear-Thinning Hyaluronic Acid Hydrogels With Secondary CrossLinking. ACS Biomater. Sci. Eng. 2, 1743-1751. doi:10.1021/ acsbiomaterials.6b00158

Ow, Z. (2021). All-Cause Failure Rates Increase With Time Following Meniscal Repair Despite Favorable Outcomes: A Systematic Review and Meta-Analysis. J. Arthrosc. Relat. Surg. 5, 00519. doi:10.1016/j.arthro.2021.05.033

Oxland, T. R. (2016). Fundamental Biomechanics of the Spine-What We Have Learned in the Past 25 Years and Future Directions. J. Biomech. 49, 817-832. doi:10.1016/j.jbiomech.2015.10.035

Ozbolat, I. T., and Hospodiuk, M. (2016). Current Advances and Future Perspectives in Extrusion-Based Bioprinting. Biomaterials. 76, 321-343. doi:10.1016/j.biomaterials.2015.10.076

Panjabi, M. M., and White, A. A. (1980). Basic Biomechanics of the Spine. Neurosurgery. 7, 76-93. doi:10.1097/00006123-198007000-00013

Park, C. K. (2015). Total Disc Replacement in Lumbar Degenerative Disc Diseases. J. Korean Neurosurg. Soc. 58, 401-411. doi:10.3340/jkns.2015.58.5.401 
Park, J. Y., Choi, J.-C., Shim, J.-H., Lee, J.-S., Park, H., Kim, S. W., et al. (2014). A Comparative Study on Collagen Type I and Hyaluronic Acid Dependent Cell Behavior for Osteochondral Tissue Bioprinting. Biofabrication. 6, 035004-035011. doi:10.1088/1758-5082/6/3/035004

Peng, Y.-Y., Dussan, D. D., and Narain, R. (2020). Thermal, Mechanical, and Electrical Properties. Polym. Sci. Nanotechnology Fundamentals Appl.. Editors R. Narain. Cambridge, MA: Elsevier, 179-201. doi:10.1016/b978-0-12-8168066.00009-1

Pham, M. H., Mehta, V. A., Tuchman, A., and Hsieh, P. C. (2015). Material Science in Cervical Total Disc Replacement. Biomed. Res. Int. 2015, 1-9. doi:10.1155/ 2015/719123

Pieri, A., Byerley, A. M., Musumeci, C. R., Salemizadehparizi, F., Vanderhorst, M. A., and Wuertz-Kozak, K. (2020). Electrospinning and 3D Bioprinting for Intervertebral Disc Tissue Engineering. Jor Spine. 3, 1-11. doi:10.1002/ jsp2.1117

Prezyna, A. P. (2000). Adverse Biologic Reactions to Polymers, Metals and Other Prosthetic Materials. Mol. Crystals Liquid Crystals Sci. Technology. Section A. Mol. Crystals Liquid Crystals. 354, 239-242. doi:10.1080/10587250008023617

Punt, I. M., Visser, V. M., Van Rhijn, L. W., Kurtz, S. M., Antonis, J., Schurink, G. W. H., et al. (2008). Complications and Reoperations of the SB Charité Lumbar Disc Prosthesis: Experience in 75 Patients. Eur. Spine J. 17, 36-43. doi:10.1007/ s00586-007-0506-8

Putzier, M., Funk, J. F., Schneider, S. V., Gross, C., Tohtz, S. W., KhodadadyanKlostermann, C., et al. (2006). Charité Total Disc Replacement-Clinical and Radiographical Results After an Average Follow-Up of 17 Years. Eur. Spine J. 15, 183-195. doi:10.1007/s00586-005-1022-3

Rajaee, S. S., Bae, H. W., Kanim, L. E. A., and Delamarter, R. B. (2012). Spinal Fusion in the United States: Analysis of Trends From 1998 to 2008. Spine. 37, 67-76. doi:10.1097/BRS.0b013e31820cccfb

Rhee, S., Puetzer, J. L., Mason, B. N., Reinhart-king, C. A., and Bonassar, L. J. (2016). 3D Bioprinting of Spatially Heterogeneous Collagen Constructs for Cartilage Tissue Engineering. ACS Biomater. Sci. Eng. 2, 1800-1805. doi:10.1021/acsbiomaterials.6b00288

Ribeiro, A., Blokzijl, M. M., Levato, R., Visser, C. W., Castilho, M., Hennink, W. E., et al. (2018). Assessing Bioink Shape Fidelity to Aid Material Development in 3D Bioprinting. Biofabrication. 10, 014102-014116. doi:10.1088/1758-5090/aa90e2

Rodts, T., Schmid, S. R., Selles, M. A., Pasang, T., and Sanchez-Caballero, S. (2019). Selective Laser Fiber Welding on Woven Polymer Fabrics for Biomedical Applications. Mater. Sci. Eng. C. 94, 628-634. doi:10.1016/j.msec.2018.10.018

Rose, S. F., Weaver, C. L., Fenwick, S. A., Horner, A., and Pawar, V. D. (2012). The Effect of Diffusion Hardened Oxidized Zirconium Wear Debris on Cell Viability and Inflammation-An In Vitro Study. J. Biomed. Mater. Res. 100B, 1359-1368. doi:10.1002/jbm.b.32704

Roseti, L., Cavallo, C., Desando, G., Parisi, V., Petretta, M., Bartolotti, I., et al. (2018). Three-Dimensional Bioprinting of Cartilage by the Use of Stem Cells: A Strategy to Improve Regeneration. Materials. 11, 1749-1820. doi:10.3390/ ma11091749

Rutz, A. L., Hyland, K. E., Jakus, A. E., Burghardt, W. R., and Shah, R. N. (2015). A Multimaterial Bioink Method for 3D Printing Tunable, Cell-Compatible Hydrogels. Adv. Mater. 27, 1607-1614. doi:10.1002/adma.201405076.A

Sabnis, A., Rahimi, M., Chapman, C., and Nguyen, K. T. (2010). Cytocompatibility Studies of an In Situ Photopolymerized Thermoresponsive Hydrogel Nanoparticle System Using Human Aortic Smooth Muscle Cells. J. Biomed. Mater. Res. A. 91, 52-59. doi:10.1002/jbm.a.32194.Cytocompatibility

Salzmann, S. N., Plais, N., Shue, J., and Girardi, F. P. (2017). Lumbar Disc Replacement Surgery-Successes and Obstacles to Widespread Adoption. Curr. Rev. Musculoskelet. Med. 10, 153-159. doi:10.1007/s12178-017-9397-4

Samandari, M., Alipanah, F., Majidzadeh-A, K., Alvarez, M. M., Trujillo-de Santiago, G., and Tamayol, A. (2021). Controlling Cellular Organization in Bioprinting through Designed 3D Microcompartmentalization. Appl. Phys. Rev. 8, 021404. doi:10.1063/5.0040732

Sánchez-Bodón, J., Ruiz-Rubio, L., Hernáez-Laviña, E., Vilas-Vilela, J. L., and Moreno-Benítez, M. I. (2021). Poly(L-lactide)-Based Anti-inflammatory Responsive Surfaces for Surgical Implants. Polymers. 13, 34-15. doi:10.3390/ polym13010034

Sansone, V. (2013). The Effects on Bone Cells of Metal Ions Released from Orthopaedic Implants. A Review. Ccmbm. 10, 34-40. doi:10.11138/ccmbm/ 2013.10.1.034
Saska, S., Pilatti, L., Blay, A., and Shibli, J. A. (2021). Bioresorbable Polymers: Advanced Materials and 4D Printing for Tissue Engineering. MDPI Polym. 13, 1-22. doi:10.3390/polym13040563

Savarino, L., Granchi, D., Ciapetti, G., Cenni, E., Greco, M., Rotini, R., et al. (2003). Ion Release in Stable Hip Arthroplasties Using Metal-On-Metal Articulating Surfaces: A Comparison between Short- and Medium-Term Results. J. Biomed. Mater. Res. 66A, 450-456. doi:10.1002/jbm.a.10595

Shankar, S., and Kesavan, D. (2016). Wear Prediction of the Lumbar Total Disc Replacement Using Finite Element Method. J. Mech. Med. Biol. 16, 1650004-1650012. doi:10.1142/S0219519416500044

Shea, M., Edwards, W. T., White, A. A., and Hayes, W. C. (1991). Variations of Stiffness and Strength Along the Human Cervical Spine. J. Biomech. 24, 95-107. doi:10.1016/0021-9290(91)90354-P

Sheikh, S. R., Thompson, N. R., Benzel, E., Steinmetz, M., Mroz, T., Tomic, D., et al. (2020). Can We Justify it? Trends in the Utilization of Spinal Fusions and Associated Reimbursement. Clin. Neurosurg. 86, E193-E202. doi:10.1093/ neuros/nyz400

Shim, J.-H., Jang, K.-M., Hahn, S. K., Park, J. Y., Jung, H., Oh, K., et al. (2016). Three-Dimensional Bioprinting of Multilayered Constructs Containing Human Mesenchymal Stromal Cells for Osteochondral Tissue Regeneration in the Rabbit Knee Joint. Biofabrication. 8, 014102. doi:10.1088/1758-5090/8/1/ 014102

Shirazi, S. F. S., Gharehkhani, S., Mehrali, M., Yarmand, H., Metselaar, H. S. C., Adib Kadri, N., et al. (2015). A Review on Powder-Based Additive Manufacturing for Tissue Engineering: Selective Laser Sintering and Inkjet 3D Printing. Sci. Technology Adv. Mater. 16, 033502-033520. doi:10.1088/ 1468-6996/16/3/033502

Singh, Y. P., Bandyopadhyay, A., and Mandal, B. B. (2019). 3D Bioprinting Using Cross-Linker-Free Silk-Gelatin Bioink for Cartilage Tissue Engineering. ACS Appl. Mater. Inter. 11, 33684-33696. doi:10.1021/ acsami.9b11644

Skardal, A., Zhang, J., and Prestwich, G. D. (2010). Bioprinting Vessel-Like Constructs Using Hyaluronan Hydrogels Crosslinked With Tetrahedral Polyethylene Glycol Tetracrylates. Biomaterials. 31, 6173-6181. doi:10.1016/ j.biomaterials.2010.04.045

Somaiah, C., Kumar, A., Mawrie, D., Sharma, A., Patil, S. D., Bhattacharyya, J., et al. (2015). Collagen Promotes Higher Adhesion, Survival and Proliferation of Mesenchymal Stem Cells. PLoS One. 10, e0145068-15. doi:10.1371/ journal.pone. 0145068

Song, X., Zhu, C., Fan, D., Mi, Y., Li, X., Fu, R., et al. (2017). A Novel Human-Like Collagen Hydrogel Scaffold With Porous Structure and Sponge-Like Properties. Polymers. 9, 638-716. doi:10.3390/polym9120638

Stemper, B. D., Board, D., Yoganandan, N., and Wolfla, C. E. (2010). Biomechanical Properties of Human Thoracic Spine Disc Segments. J. Craniovertebr Junction Spine. 1, 18-22. doi:10.4103/0974-8237.65477

Stevens, L. R., Gilmore, K. J., Wallace, G. G., and in het Panhuis, M. (2016). Tissue Engineering With Gellan Gum. Biomater. Sci. 4, 1276-1290. doi:10.1039/ c6bm00322b

Storey, D. M., and Webster, T. J. (2014). Novel Nano-Rough Polymers for Cartilage Tissue Engineering. Int. J. Nano. 9, 1845-1853.

Sun, B., Lian, M., Han, Y., Mo, X., Jiang, W., Qiao, Z., et al. (2021a). A 3DBioprinted Dual Growth Factor-Releasing Intervertebral Disc Scaffold Induces Nucleus Pulposus and Annulus Fibrosus Reconstruction. Bioactive Mater. 6, 179-190. doi:10.1016/j.bioactmat.2020.06.022

Sun, Y., Zhang, Y., Wu, Q., Gao, F., Wei, Y., Ma, Y., et al. (2021b). 3D-Bioprinting Ready-To-Implant Anisotropic Menisci Recapitulate Healthy Meniscus Phenotype and Prevent Secondary Joint Degeneration. Theranostics. 11, 5160-5173. doi:10.7150/thno.54864

Sun, Y., You, Y., Jiang, W., Wang, B., Wu, Q., and Dai, K. (2020a). 3D Bioprinting Dual-Factor Releasing and Gradient-Structured Constructs Ready to Implant for Anisotropic Cartilage Regeneration. Sci. Adv. 6, eaay1422-10. doi:10.1126/ sciadv.aay 1422

Sun, Y., You, Y., Jiang, W., Wu, Q., Wang, B., and Dai, K. (2020b). Generating Ready-To-Implant Anisotropic Menisci by 3D-Bioprinting Protein-Releasing Cell-Laden Hydrogel-Polymer Composite Scaffold. Appl. Mater. Today. 18, 100469. doi:10.1016/j.apmt.2019.100469

Sun, Y., You, Y., Jiang, W., Zhai, Z., and Dai, K. (2019). 3D-bioprinting a Genetically Inspired Cartilage Scaffold With GDF5-Conjugated BMSC- 
Laden Hydrogel and Polymer for Cartilage Repair. Theranostics. 9, 6949-6961. doi:10.7150/thno.38061

Szymański, T. (2020). Utilization of Carbon Nanotubes in Manufacturing of 3D Cartilage and Bone Scaffolds. Materials (Basel). 13, 1-25.

Tappa, K., and Jammalamadaka, U. (2018). Novel Biomaterials Used in Medical 3D Printing Techniques. J. Funct. Biomater. 9, 17. doi:10.3390/jfb9010017

Tavakoli, J., Diwan, A. D., and Tipper, J. L. (2020). Advanced Strategies for the Regeneration of Lumbar Disc Annulus Fibrosus. Int. J. Mol. Sci. 21, 4889-4920. doi:10.3390/ijms21144889

Thompson, W. O., Thaete, F. L., Fu, F. H., and Dye, S. F. (1991). Tibial Meniscal Dynamics Using Three-Dimensional Reconstruction of Magnetic Resonance Images. Am. J. Sports Med. 19, 210-216. doi:10.1177/ 036354659101900302

Urban, R. M., Jacobs, J. J., Tomlinson, M. J., Gavrilovic, J., Black, J., and Peoc'H, M. (2000). Dissemination of Wear Particles to the Liver, Spleen, and Abdominal Lymph Nodes of Patients With Hip or Knee Replacement ${ }^{*}$. The J. Bone Jt. Surgery-American. 82, 457-477. doi:10.2106/00004623-200004000-00002

U.S Food and Drug Administration (2015). Center for Devices and Radiological Health. MD: Silver Spring. Available at: https://www.accessdata.fda.gov/cdrh_ docs/pdf18/K181644.pdf.

U.S. Food and Drug Administration (2020). Center for Devices and Radiological Health. MD: Silver Spring. Available at: https://www.accessdata.fda.gov/cdrh_ docs/pdf5/P050010S020A.pdf.

Vaz, R. A., Xavier, P., Brito, S., Dantas, J., Duque, S., Consciência, J. G., et al. (2019). Metallosis: A New Form of Autoimmune/Autoinflammatory Syndrome Induced by Adjuvants Syndrome (ASIA)? Eur. J. Case Rep. Intern. Med. Met. 6, 1-3. doi:10.12890/201910.12890/2019_001034

Vernengo, A. J., Grad, S., Eglin, D., Alini, M., and Li, Z. (2020). Bioprinting Tissue Analogues With Decellularized Extracellular Matrix Bioink for Regeneration and Tissue Models of Cartilage and Intervertebral Discs. Adv. Funct. Mater. 30, 1909044. doi:10.1002/adfm.201909044

Veronesi, F., Di Matteo, B., Vitale, N. D., Filardo, G., Visani, A., Kon, E., et al. (2021). Biosynthetic Scaffolds for Partial Meniscal Loss: A Systematic Review From Animal Models to Clinical Practice. Bioactive Mater. 6, 3782-3800. doi:10.1016/j.bioactmat.2021.03.033

Wei, W., Ma, Y., Yao, X., Zhou, W., Wang, X., Li, C., et al. (2021). Advanced Hydrogels for the Repair of Cartilage Defects and Regeneration. Bioactive Mater. 6, 998-1011. doi:10.1016/j.bioactmat.2020.09.030

Wilke, H. J., Neef, P., Caimi, M., Hoogland, T., and Claes, L. E. (1999). New In Vivo Measurements of Pressures in the Intervertebral Disc in Daily Life. Spine. 24, 755-762. doi:10.1097/00007632-199904150-00005

Wu, D., Tan, J., Yao, L., Tian, J., Luo, B., Li, L., et al. (2021). Customized Composite Intervertebral Disc Scaffolds by Integrated 3D Bioprinting for Therapeutic Implantation. Composites A: Appl. Sci. Manufacturing. 147, 106468-106511. doi:10.1016/j.compositesa.2021.106468

Wu, D., Yu, Y., Tan, J., Huang, L., Luo, B., Lu, L., et al. (2018). 3D Bioprinting of Gellan Gum and Poly (Ethylene Glycol) Diacrylate Based Hydrogels to Produce Human-Scale Constructs with High-Fidelity. Mater. Des. 160, 486-495. doi:10.1016/j.matdes.2018.09.040

Wu, W., Deconinck, A., and Lewis, J. A. (2011). Omnidirectional Printing of 3D Microvascular Networks. Adv. Mater. 23, H178-H183. doi:10.1002/ adma. 201004625

Yanke, A., and Cole, B. (2019) Editor. Joint Preservation of the Knee: A Clinical Casebook. Cham, Switzerland: Springer, 1-8.

Yang, F., Zhao, J., Koshut, W. J., Watt, J., Riboh, J. C., Gall, K., et al. (2020a). A Synthetic Hydrogel Composite With the Mechanical Behavior and Durability of
Cartilage. Adv. Funct. Mater. 30, 2003451-2003458. doi:10.1002/ adfm.202003451

Yang, Y., Yang, G., Song, Y., Xu, Y., Zhao, S., and Zhang, W. (2020b). 3D Bioprinted Integrated Osteochondral Scaffold-Mediated Repair of Articular Cartilage Defects in the Rabbit Knee. J. Med. Biol. Eng. 40, 71-81. doi:10.1007/ s40846-019-00481-y

Yang, Z., Zhao, T., Gao, C., Cao, F., Li, H., Liao, Z., et al. (2021). 3DBioprinted Difunctional Scaffold for In Situ Cartilage Regeneration Based on Aptamer-Directed Cell Recruitment and Growth Factor-Enhanced Cell Chondrogenesis. ACS Appl. Mater. Inter. 13, 23369-23383. doi:10.1021/ acsami.1c01844

Yoganandan, N., Sances, A., and Pintar, F. (1989). Biomechanical Evaluation of the Axial Compressive Responses of the Human Cadaveric and Manikin Necks. J. Biomech. Eng. 111, 250-255. doi:10.1115/1.3168374

Yu, Y., Zhang, Y., Martin, J. A., and Ozbolat, I. T. (2013). Evaluation of Cell Viability and Functionality in Vessel-Like Bioprintable Cell-Laden Tubular Channels. J. Biomech. Eng. 135, 1-9. doi:10.1115/1.4024575

Yup Lee, J., and Kim, S.-Y. (2010). Alumina-on-Polyethylene Bearing Surfaces in Total Hip Arthroplasty. Toorthj. 4, 56-60. doi:10.2174/ 1874325001004010056

Zhang, L., Hu, J., and Athanasiou, K. A. (2009). The Role of Tissue Engineering in Articular Cartilage Repair and Regeneration. Crit. Rev. Biomed. Eng. 37, 1-57. doi:10.1615/CritRevBiomedEng.v37.i1-2.10

Zhang, Q., Lu, H., Kawazoe, N., and Chen, G. (2014). Pore Size Effect of Collagen Scaffolds on Cartilage Regeneration. Acta Biomater. 10, 2005-2013. doi:10.1016/j.actbio.2013.12.042

Zhang, Y., Yu, J., Ren, K., Zuo, J., Ding, J., and Chen, X. (2019). Thermosensitive Hydrogels as Scaffolds for Cartilage Tissue Engineering. Biomacromolecules. 20, 1478-1492. doi:10.1021/acs.biomac.9b00043

Zhu, S., Chen, P., Chen, Y., Li, M., Chen, C., and Lu, H. (2020). 3D-Printed Extracellular Matrix/Polyethylene Glycol Diacrylate Hydrogel Incorporating the Anti-Inflammatory Phytomolecule Honokiol for Regeneration of Osteochondral Defects. Am. J. Sports Med. 48, 2808-2818. doi:10.1177/ 0363546520941842

Zou, A. H., Feng, J. E., Novikov, D., O'Connor, C. M., Anoushiravani, A. A., Schwarzkopf, R., et al. (2020). Are Oxinium Femoral Heads Superior to Alternative Bearing Surface Materials? A Systematic Review. J. Hip Surg. 4, 142-148. doi:10.1055/s-0040-1718506

Conflict of Interest: The authors declare that the research was conducted in the absence of any commercial or financial relationships that could be construed as a potential conflict of interest.

Publisher's Note: All claims expressed in this article are solely those of the authors and do not necessarily represent those of their affiliated organizations, or those of the publisher, the editors and the reviewers. Any product that may be evaluated in this article, or claim that may be made by its manufacturer, is not guaranteed or endorsed by the publisher.

Copyright $\odot 2021$ Perera, Ivone, Natekin, Wilga, Shen and Menon. This is an openaccess article distributed under the terms of the Creative Commons Attribution License (CC BY). The use, distribution or reproduction in other forums is permitted, provided the original author(s) and the copyright owner(s) are credited and that the original publication in this journal is cited, in accordance with accepted academic practice. No use, distribution or reproduction is permitted which does not comply with these terms. 